\title{
Pharmacotherapy of osteoporosis
}

Republishes with permision from the SOUTH AFRICAN CLINICAL GUIDELINE FOR THE DIAGNOSIS AND MANAGEMENT OF OSTEOPOROSIS: 2017. For the National Osteoporosis Foundation of South Africa (NOFSA). Chapter 11:103-145

The pharmacological agents currently used in the management of osteoporosis include:

- Drugs which specifically aim to prevent bone loss, improve bone strength and reduce the risk of future fractures (i.e. specific therapeutic agents).

- Drugs which reduce the pain and disability associated with a fracture.

The administration of specific therapeutic agents will not result in short-term symptomatic pain relief, and vice versa. Although exceptions to this rule may exist (see 13.4), this must be explained to patients if long-term compliance is to be ensured. Specific therapeutic agents used in osteoporosis are usually classified as inhibitors of bone resorption (anticatabolics), stimulators of bone formation (anabolics), or dual action agents (Table IX).

Bone is a dynamic tissue, continuously engaged in maintenance remodelling. At any point in time, some bone will have been resorbed, yet not replaced. This is referred to as the remodelling space, and is increased in osteoporosis. ARAs decrease the rate of initiation of new remodelling cycles, resulting in fewer remodelling sites and a decrease in the remodelling space.

Table IX: Drugs currently used to treat osteoporosis

\section{Inhibitors of bone resorption: anticatabolics}

Calcium/vitamin D
Estrogen/progestins
Estrogen analogues, selective estrogen receptor modulators (SERMS),
testosterone
- $\quad$ raloxifene
- tibolone
- $\quad$ phyto-estrogens
- testosterone
Bisphosphonates
- $\quad$ alendronate
- ibandronate
- $\quad$ risedronate
- zoledronate
Calcitonins

\section{Stimulators of bone formation: anabolics}

\section{Parathyroid hormone}

Drugs with dual and/or complex actions on bone

\section{Strontium ranelate}

Vitamin D metabolites (calcitriol/alfacalcidol)

Anabolic steroids

Thiazide diuretics/indapamide

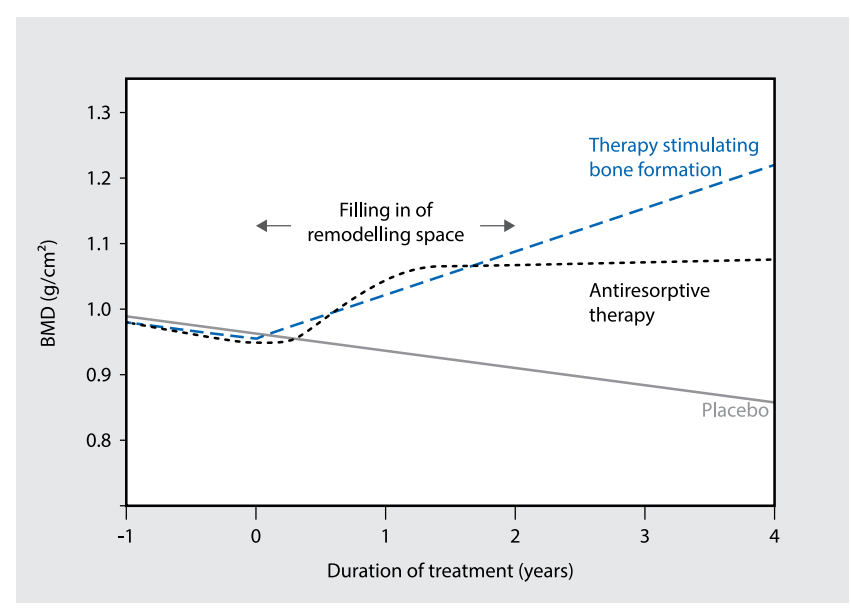

Figure 7: Effects on BMD after treatment with ARAs and bone formation-stimulating drugs.

Adapted from Parfitt305 and Eastell.306

ARAs aim to stabilise BMD and prevent further loss of bone. After initiating treatment with ARAs, bone resorption rapidly decreases while formation continues, resulting in the filling in of the remodelling space and a modest (2-8\%) increase in BMD over this time. Coupling between resorption and formation, however, results in a subsequent decrease in the rate of formation and, after two to three years, bone mass usually stabilises for the duration of treatment (Figure 7). ARAs, like the bisphosphonates and estrogen, do not only decrease the rate of bone loss, but also decrease the depth of resorption cavities and, therefore, improve bone quality. Only drugs which directly stimulate bone formation can, however, be expected to result in a sustained increase in BMD and correction of micro-architecture.

The choice of a specific agent to treat osteoporosis is made difficult by the lack of head-to-head studies comparing the efficacy and safety of different agents. No "best drug" scenario therefore exists, and such a choice usually has to take into account the patient profile (age, gender, general health), the nature of the disease (cause, severity, skeletal sites involved) and the availability and side effects of specific drugs.

\subsection{Inhibitors of bone resorption}

Inhibition of bone resorption can be effected by manipulation of (i) osteoclast formation, (ii) activity of mature osteoclasts, 
and (iii) osteoclast apoptosis. Currently available ARAs include calcium/vitamin D, estrogens and SERMS which largely, although not exclusively, suppress osteoclastogenesis, and the bisphosphonates, which inhibit osteoclast activity and promote osteoclast apoptosis. Improved understanding of osteoclast biology has recently uncovered a number of novel targets for controlling osteoclast formation and activity. Since some of these new drugs will be launched within the next year or two, it is appropriate to mention them here, although any discussion of these agents falls outside the scope of this guideline. Some of these newly developed drugs include inhibitors of the RANKL pathway like the monoclonal antibody denosumab, which inhibits osteoclastogenesis and has already been shown to increase BMD and to reduce fracture risk. ${ }^{307}$ Inhibitors of osteoclast activity include the cathepsin $\mathrm{K}$ protease inhibitor odanacatib, inhibitors of carbonic anhydrase (CA2), osteoclast integrins, vacuolar $\mathrm{H}^{+}$-ATPase, and various signalling pathways (p38 kinase, p60 C-SRC kinase), as well as glucagon-like peptide 2 which inhibits the circadian, nocturnal rise in bone resorption. ${ }^{308-310}$

\subsubsection{Calcium and vitamin D}

The more common causes of calcium and vitamin D deficiency (see 7.1.9), the assessment of calcium and vitamin $D$ (measured as 25OHD) status (see 9.1), and the effects of calcium and vitamin $\mathrm{D}$ on peak bone mass, age-related bone loss and fracture risk (10.1.1.1) have been discussed in some detail. We shall now briefly comment on pharmacological supplementation of calcium and vitamin D.

\subsubsection{Calcium supplementation}

An adequate intake of calcium has been advocated by health care professionals for many decades. Following the publication, by Mark Bolland et al. in 2010311, of an association between calcium supplementation and the risk of myocardial infarction, as well as the article by $\mathrm{Li}$ et al published in $2012^{312}$ supporting some of these findings, a group of academic and industry experts in the fields of nutrition, cardiology, epidemiology, food science, bone health and integrative medicine, examined all the available data and in November 2012 Robert Heaney and co-workers published their findings in the journal Advances in Nutrition. ${ }^{313}$

It is known that calcium and vitamin D supplementation may be useful in reducing the risk of osteoporosis in postmenopausal women when dietary intakes of these nutrients are low. The Food and Nutrition Board of the US Institute of Medicine (IOM) currently designates an upper calcium-intake level of $2500 \mathrm{mg} / \mathrm{d}$ for adults aged 19-50y, and 2000mg/day for individuals over the age of $50 y$, as being free of risk of adverse health effects. ${ }^{209}$ Consumption of calcium and vitamin D-containing supplements has increased in recent years, as determined by the 2003-2006 NHANES report. ${ }^{314}$ This study showed mean intakes of calcium from supplements, of $578 \mathrm{mg} / \mathrm{d}$ and $608 \mathrm{mg} / \mathrm{d}$ for women 51 $70 \mathrm{y}$ and over 71y respectively. Prior to a series of reports from Bolland et $\mathrm{al}^{311,315,316}$, Reid et $\mathrm{al}^{317}$ and Li et al $\mathbf{l}^{312}$, no suggestions of serious side-effects from these supplemental calcium doses were reported. Bolland et al. then reported that the use of calcium supplements, with or without added vitamin D, modestly increased cardiovascular risk. More recently a publication by Li et al reported similar findings. These reports have received wide publicity and have resulted in much confusion and concern amongst patients and doctors alike, even prompting the revision of the recommended intakes for calcium supplement use.

Subsequently, Heaney and co-workers ${ }^{313}$ reviewed data from 16 studies, involving more than 358000 individuals and concluded that there was no association between calcium intake and atherosclerotic heart disease or stroke. Although a few of the studies showed a weak, albeit statistically significant positive association, a similar number showed the converse, namely a decreased risk of cardiovascular disease (CVD) Heaney and co-workers therefore suggest that the evidence presented to date regarding the postulated relationship between the use of calcium supplementation and the increased risk of CVD, is insufficient to warrant changing the IOM recommendations for calcium supplementation in those with inadequate dietary intakes. It is also important to remember that CVD was not the primary outcome in most of these studies and some of the reports suggested a bias for cardiovascular events in the calcium supplementation studies because patients self-reported these cardiovascular events. ${ }^{318}$ After reviewing the available evidence, Heaney et al conclude that there is currently too little evidence to substantiate a causal relationship between calcium supplementation and an increased risk of cardiovascular events and call for better designed prospective studies to specifically evaluate risks and benefits of calcium supplementation (with/ without vitamin D).

1. It is important to remember that, when supplements are used, the yield of elemental calcium varies with the calcium salt employed (Table X). Calcium carbonate preparations should be taken with meals, since $\mathrm{HCl}$ is required to liberate free calcium and improve its intestinal absorption. Although differences have been reported in the bioavailability of calcium between proprietary preparations, these are usually small and probably not clinically significant. If concomitant deficiencies in vitamin D (see 7.1.9) or magnesium (e.g. alcoholism, diabetes, malabsorption syndromes, use of diuretics), known to impair calcium bioavailability, are present, they should be supplemented. The routine supplementation of magnesium, either alone or in combination, is not recommended. Calcium is easy to use and generally well tolerated, although patient compliance is often poor because of gastrointestinal side effects, particularly constipation.

Table X: Elemental calcium content of commonly used calcium supplements

\begin{tabular}{lc}
\hline \multicolumn{1}{c}{ Calcium salt } & Yield of elemental calcium (\%) \\
\hline Calcium carbonate & 40 \\
Tribasic calcium phosphate & 38 \\
Calcium chloride & 27 \\
Dolomite & 22 \\
Calcium citrate & 21 \\
Calcium lactate & 13 \\
Calcium gluconate & 9 \\
\hline
\end{tabular}




\section{What does NOFSA recommend?}

- An adequate daily calcium intake of approximately $1000 \mathrm{mg} /$ day is important for normal bone health. This should preferably be obtained from the diet of which low fat/fat free dairy would be an important source.

- We know that more than $50 \%$ of individuals do not reach this intake through diet alone - NOFSA therefore supports calcium supplementation of $500-600 \mathrm{mg} /$ day as acceptable and safe.

- High dose calcium supplementation in patients already consuming ample dairy, and especially those with known kidney failure or CVD, is unnecessary and should be avoided.

\subsubsection{Vitamin D supplementation}

Parfitt has defined three degrees of hypovitaminosis $D$ osteopathy. ${ }^{319}$ Stage 1 is characterised by diminished intestinal calcium absorption, which results in osteoporosis without histologic changes of the skeleton; stage 2 , by impaired calcium absorption and osteoporosis, plus early histologic features of osteomalacia without any laboratory abnormalities suggestive of osteomalacia; and stage 3, by clinical and laboratory features of osteomalacia. Prophylactic doses of vitamin $D$ range from 800-1 000 IU per day, and either cholecalciferol or ergocalciferol may be used (see 10.1.1.1). If serum 250HD levels indicate relative vitamin $D$ insufficiency $(<20 \mathrm{ng} / \mathrm{ml}$ or $50 \mathrm{nmol} / \mathrm{l})$, serum PTH levels and bone turnover start to increase, high-turnover osteoporosis develops. Circulating 25OHD levels > $10 \mathrm{ng} / \mathrm{ml}$ appear quite adequate to prevent osteomalacia from developing, whereas levels below $5-8 \mathrm{ng} / \mathrm{ml}$ are usually accompanied by a mineralisation defect. If osteomalacia is present, treatment with 50000 IU per week is indicated. There are no indications for or advantages in using one of the more potent vitamin D metabolites or analogues for treating simple nutritional vitamin D deficiency, although these agents should be considered if the vitamin $D$ deficiency has resulted from intestinal malabsorption. ${ }^{320}$ When larger doses of vitamin $\mathrm{D}$ are given, monitoring of urinary calcium is usually recommended, although intakes of up to 4,000 IU per day have been shown to be well tolerated without abnormal increases in serum or urine calcium. ${ }^{245,321,322}$ Doses of 50,000 IU Vitamin D3 administered weekly have been advocated for treatment of Vitamin D deficiency $(<12 \mathrm{ng} / \mathrm{ml}$ or $30 \mathrm{nmol} / \mathrm{l})$. There is however insufficient data available for us to make firm recommendations in this regard.

\subsubsection{Hormone therapy (HT)}

In this guideline, the term is used generically to denote the use of estrogen \pm progestin in postmenopausal women. Estrogen alone is referred to as ET, and estrogen in combination with progestogen as EPT.

Until 2002, HT was regarded as first-line therapy for the prevention and treatment of postmenopausal osteoporosis. Not only was HT thought to be highly effective in the alleviation of the symptoms of the menopause, but numerous observational studies suggested that it also significantly reduced the risk of coronary heart disease (CHD) by $30-50 \%$, and that of cerebrovascular accidents by $15-20 \% .323-326$ It was, therefore, reasoned that any risks associated with HT were far outweighed by these extraskeletal benefits. However, in 1998, the first RCT of oestrogen plus progestin for secondary prevention of coronary heart disease in postmenopausal women (Heart and Estrogen/ progestin Replacement Study, HERS) not only failed to document a protective effect, but reported a significantly higher rate of $\mathrm{CHD}$ events during the first year of HT. ${ }^{327}$ In 2002, the first results of the WHI were published, confirming a beneficial effect of $\mathrm{HT}$ on fracture prevention, but failing to show any benefits of HT on CHD and, in fact, reporting a $29 \%$ increase in the risk of non-fatal CHD events, although this did not reach statistical significance. ${ }^{328}$ The risk of stroke was also increased and this did reach nominal, but not adjusted, statistical significance. These observations resulted in regulatory bodies like the American Food and Drug Administration (FDA) and the European Medicines Agency (EMEA) advising against the use of HT as first-line therapy for the management of osteoporosis. During the past eight years, much debate, reassessment of data and some new information have provided new insights which will be summarised here, since the subject has been extensively reviewed, both internationally ${ }^{329-331}$ and locally. ${ }^{332,333}$

\subsubsection{Effects of HT on bone}

Numerous observational and controlled studies have provided evidence of a beneficial effect of $\mathrm{HT}$ on BMD and vertebral fracture risk, but we had to wait for the WHI to convincingly document a reduction in the rate of all osteoporosis-related fractures, including hip fractures.

\subsection{Bone mineral density}

$\mathrm{HT}$ has consistently been shown to increase BMD at all major skeletal sites. ${ }^{34-339}$ In 2001, a meta-analysis of 57 RCTs found an average increase of BMD of $6.8 \%$ at the spine and $4.1 \%$ at the femoral neck after two years of $\mathrm{HT} .{ }^{336}$ Similar data were reported in the Postmenopausal Estrogen/Progestin Intervention (PEPI), ${ }^{337}$ the National Osteoporosis Risk Assessment (NORA), ${ }^{338}$ and the WHI. ${ }^{339}$ The response is dose related, and a daily dose of $0.625 \mathrm{mg}$ conjugated equine estrogen (CE) (equivalents are $2 \mathrm{mg}$ estradiol valerate; $1 \mathrm{mg}$ micronised 17- $\beta$-estradiol; $50 \mu \mathrm{g}$ estradiol transdermally) is required for an optimal effect. Higher doses do not appear to result in an improvement, although unconfirmed reports have suggested that higher doses may have an anabolic effect on bone and may be required if predominantly cortical osteopenia is treated. Given the extraskeletal side effects of HT (see below), much interest resides in the effects of low-dose HT on bone. RCTs using half the conventional HT dose (oral $0.3 \mathrm{mg}$ $\mathrm{CE} /$ day; oral $0.5 \mathrm{mg}$ micronised $17-\beta$-estradiol/day; transdermal $25 \mu \mathrm{g}$ estradiol/day) have shown significant increases in both spine (2-4.7\%) and hip (1-3.6\%) BMD. ${ }^{340-342}$ In fact, an ultra-low oral dose of $0.25 \mathrm{mg}$ micronised estradiol per day significantly suppressed biochemical markers of bone turnover and increased vertebral and hip BMD by $2-4 \%{ }^{343}$ The beneficial effects of lowdose HT on BMD appear to be most pronounced when ample calcium and vitamin $D$ is supplied, and an additive effect of these agents on bone resorption has been suggested. ${ }^{340,341}$ Optimal vitamin $\mathrm{D}$ repletion has also recently been shown to augment 
the BMD and antifracture efficacy of the bisphosphonates and raloxifene, and seems to be necessary to maximise the bone effects of most ARAs. ${ }^{344}$ Significant lumbar spine and hip BMD improvements have also been noted with systemic estrogen doses delivered via a vaginal ring (Femring ${ }^{\circledR}$, Menoring $\left.{ }^{\circledR}\right) .^{345}$

The route of administration (tablet, skin implant, patch, gel, nasal spray) appears to be relatively unimportant as far as bone is concerned, but impacts significantly on the extraskeletal effects of HT (see below). The bone-sparing effect of estrogen persists as long as therapy is given. It is most pronounced during the first five to 10 years after the menopause. Some evidence suggests that catch-up bone loss occurs after HT has been discontinued, while other studies show that the rate of bone loss after HT is stopped is similar to the rate immediately before therapy was instituted. Although the PERF study ${ }^{346}$ suggested that two to three years of HT have long-term protective effects on bone loss and osteoporotic fractures, the larger NORA ${ }^{338}$ and Million Women Study ${ }^{347}$ strongly supported the contention that bone is rapidly lost once $\mathrm{HT}$ is discontinued. If bone protection is required at this stage, the addition of a bisphosphonate or other boneactive drug is, therefore, recommended. ${ }^{348,349}$ Similar to other ARAs, $\mathrm{HT}$ often results in a modest transient increase in BMD. This can be ascribed to filling in of the remodelling space (see Figure 5), is more evident if pretreatment bone turnover is high, ${ }^{168}$ and is usually not sustained beyond two to three years. Not all patients respond to HT and some $10-20 \%$ of subjects lose bone at conventional doses of estrogen. It is unclear whether this is the result of poor adherence to treatment or inherent resistance to the action of the hormone. The antiresorptive mechanism of action of estrogens (largely inhibition of osteoclastogenesis) differs from that of the bisphosphonates (predominantly suppression of osteoclast activity), and preliminary studies have suggested additive effects on bone markers and BMD when these agents are used in combination. ${ }^{350,351}$ No data on fracture risk are, however, available.

When EPT is given, the choice of progestin may also influence the skeletal response. Progestins which possess greater androgenic and lesser glucocorticoid activity (e.g. norethisterone acetate, NETA) have been shown to exibit superior protection against bone loss as well as fracture, compared with progestins with less androgenic or more glucocorticoid activity (e.g. medroxyprogesterone acetate, MPA). ${ }^{352}$ Further studies are, however, required to confirm this observation, which has been refuted by some. ${ }^{347}$ No current evidence supports the contention that progestins alone improve BMD or decrease fracture risk.

\subsection{Fractures}

Prior to the publication of the WHI in 2002, evidence from observational studies and some RCTs indicated that standarddose HT reduced the risk of osteoporotic fractures. ${ }^{334-336,353-357}$ Evidence was convincing for a reduction in the risk of vertebral fractures, but less so for hip fracture, although two metaanalyses of RCTs did report a reduction in nonvertebral fractures of $27 \% .{ }^{323,358}$ The WHI was, however, the landmark study which unequivocally proved that both ET and EPT reduced the risk of spine, hip and total fractures by $24-39 \% .{ }^{328,339,359}$ Subsequently, these results were corroborated by two large observational studies: NORA, which is a longitudinal follow-up study of 200160 postmenopausal women, ${ }^{338}$ and the Million Women's Study, a prospective observational study of 138737 postmenopausal females. ${ }^{347}$

The large $\mathrm{WHI}(\mathrm{n}=26,600)$ study was unique in a number of ways, and redefined the role of $\mathrm{HT}$ in the management of osteoporosis. It was not only the first RCT to unequivocally prove that HT reduced the risk of all major osteoporotic fractures but, unlike other osteoporosis trials, patients were unselected and not at high fracture risk. Most patients did not have a BMD in the osteoporosis range, yet HT also proved to be effective in those subjects with osteopenia. Furthermore, study outcomes did not include spine X-rays, so that the effects of HT on radiological fractures were undoubtedly underestimated. We have previously taken cognisance of the fact that lower doses of HT improve BMD and suppress biochemical markers of bone turnover. Unfortunately, no study to date has yielded data to support the theory that low-dose HT affords protection against fractures.

\subsubsection{Non-skeletal effects of HT}

The therapeutic envelope of any intervention is defined by the ratio of benefit to risk. The WHI report clearly highlighted the major benefits of HT for bone health and fracture prevention.

The non- skeletal effects of HT, emanating from the WHI and other studies, $323,327-333,337,338,360$ can be tabulated as follows:

- Systemic HT is the only treatment which has been consistently shown to be superior to placebo in the treatment of vasomotor symptoms and the associated sleep disorders that attend the early menopause.

- Systemic or local HT is effective in the management of vulval and vaginal atrophy (VVA)

- EPT is associated with a small, but significant, increase in the risk of invasive breast cancer if used for longer than seven years. Although the relative risk is in the order of 1.35 , the absolute increase in risk is small (less than $0.1 \%$ per year). ET does not increase the risk of breast cancer and may, in fact, reduce such risk if used for less than 7 years.

- The risk of venous thromboembolism (VTE) is doubled with HT. The effect is maximal in the first year of treatment and more pronounced with advancing age, obesity, and previous VTE. The absolute risk of VTE in the age group 50-60 years is very small (approximately 2/1 000 per year). Nonetheless, it would seem prudent not to recommend HT in patients with spontaneous thrombosis, particularly those occurring during pregnancy or whilst taking the estrogen contraceptive pill, unless abnormalities of coagulation and fibrinolysis have been excluded. It is also advisable to discontinue HT temporarily during surgery or immobilisation (including air travel).

- The WHI study failed to demonstrate a reduced risk of CHD in HT users. In the EPT arm, a non-significant increase (7/10 000 women per year) in non-fatal CHD was, in fact, found. The WHI study subjects were, however, relatively old (average 
age 64 years) and not screened for CHD risk factors. Recently, the "therapeutic window" hypothesis has evolved, which attempts to accommodate the disparate observations that HT may be protective against CHD under certain circumstances, yet may exacerbate the risk of CHD in other circumstances. According to this hypothesis, which is supported by substantial basic and epidemiological data, estrogen may offer protection when the arterial endothelium is still healthy and intact. In elderly women with established vascular disease, estrogen may, however, destabilise an atherosclerotic plaque, and precipitate an event. Data from the ET arm of the WHI have documented a significant trend $(p=0.02)$ for CHD events to be lower the shorter the period since the menopause, as well as in younger individuals. ${ }^{361}$ Even more encouraging results have emanated from a recent analysis of the Nurses' Health Study ${ }^{362}$ in women starting HT near the menopause, showing a significantly reduced risk of $\mathrm{CHD}$ for both women on estrogen alone $(\mathrm{RR}=0.66 ; 95 \% \mathrm{Cl} 0.54-0.80)$ and for women on estrogen plus progestogen ( $\mathrm{RR}=0.72 ; 95 \% \mathrm{Cl} 0.56-0.92)$. A recent subanalysis of subjects in the ET arm of the WHI study, which assessed the coronary artery calcium scores (CACS) in 1064 women aged 50-59 years, concluded that the calcified plaque burden was significantly lower in those who had been on $\mathrm{ET}^{363}$

- The WHI study reported an increased risk of stroke in both ET (HR 1.39) and EPT (HR 1.39) users (significant on unadjusted, but not on adjusted values), which is consistent with results from the Nurses' Health Study. Unlike VTE, the effect was not confined to the first year of HT, but was maintained throughout the study. These results are to be contrasted with those of the Danish Nurses Study ${ }^{364}$ which found that unopposed estradiol (1 mg per day) was not associated with an increased risk of stroke (HR 0.80; Cl: 0.40-1.61) in 13122 healthy postmenopausal women followed up for five years. It has been suggested that the effect of HT on stroke may be dose-related, with smaller doses being protective and larger doses harmful. 365

- The risk of endometrial cancer is significantly increased (two- to fivefold) in women who use estrogen without added progestin. It is, therefore, mandatory that all women with an intact uterus, who wish to use HT, add a progestin to the estrogen regimen. EPT largely eliminates the increased risk, provided that the dose and schedule of progestogen therapy is adequate to prevent endometrial hyperplasia (e.g. $5 \mathrm{mg}$ MPA, $2 \mathrm{mg}$ NETA or $10 \mathrm{mg}$ Dydrogesterone for 14 days per month or $2.5 \mathrm{mg}$ MPA, $1 \mathrm{mg}$ NETA or $5 \mathrm{mg}$ dydrogesterone per day continuously).

- The WHI study failed to demonstrate a beneficial effect of HT on cognition - Worsening of cognitive function may, in fact, occur if initiated in patients over the age of 65 years. EPT reduces the risk of colorectal cancer. In the elderly, observational studies have suggested that HT may decrease the propensity to falling, but this has not been confirmed in RCTs, including the WHI.

- Occasionally, HT-induced exacerbations of hypertension, migraine, gall bladder disease, endometriosis, porphyria, systemic lupus erythematosus or deterioration in diabetes control may occur. Melanoma has been reported to recur during pregnancy and, for this reason, HT is usually avoided. Contraindications for HT are listed in Table XI.

- "Less serious", often transient, side effects of HT commonly occur during the first year of therapy and constitute one of the principal reasons why more than $50 \%$ of women stop taking treatment during this time. Counselling and sympathetic attention to unwelcome menstrual bleeds, breast discomfort, fluid retention, mood changes and weight gain (despite reports to the contrary) are paramount to ensure successful $\mathrm{HT}$ and a satisfied patient.

\subsection{Oral preparations}

In hysterectomised women, only estrogen preparations are required and this may be given continuously. In women with a uterus, combined HT (EPT) using an estrogen plus progestogen is mandatory. The following regimens may be utilised:

i. Sequentially opposed HT. Estrogen is given for 21-28 days, and the progestogen for the last 10-14 days. Various monthly packs of an estrogen plus progestin are also available. After the progestogen is stopped, women experience a withdrawal bleed, but obviously this does not imply a return of fertility. Unfortunately, the return of monthly periods is one of the most common reasons for non-compliance with $\mathrm{HT}$.

ii. Continuous combined HT. Both estrogen and progestogen are given continuously, either individually or as a single oral preparation. Should EPT be required for more than five years, it is recommended to convert from sequential to continuous combined HT. Continuous progestogen administration requires a minimum of $2.5 \mathrm{mg}$ MPA, $5 \mathrm{mg}$ dydrogesterone or $1 \mathrm{mg}$ NETA daily to suppress endometrial proliferation and generally does not induce cyclical bleeding. Unpredictable bleeding or spotting occurs in some $25 \%$ of women during the first six months, although more than $80 \%$ of subjects have no bleeding after one year. Irregular bleeds occur more often in perimenopausal women than in women with a longer duration of menopause. Irregular bleeding may be minimised by increasing the dose of progestogen. Bleeding which persists beyond the first six months require further gynaecological assessment.

iii. Long cycle sequentially opposed HT. Here the progestin is given for 14 days every three months. Less extensive data on endometrial protection are available with this regimen, and it is generally not recommended on present evidence.

\subsection{Non-oral preparations}

Non-oral routes of HT administration (e.g. transdermal) avoid the first-pass effect on the liver and are preferable in hypertriglyceridaemia, carbohydrate intolerance, obesity, mild liver disease, gallstones, previous DVT, and in smokers. ${ }^{353,361,367}$

\subsubsection{The perimenopause}

The perimenopause is generally defined as that period preceding the menopause during which periods of menstrual irregularities 
Table XI: Contraindications to $\mathrm{HT}^{332,366}$

\begin{tabular}{ll}
\hline - & Kurrent, past or suspected breast cancer \\
- & Undiagnosed genital bleeding \\
- & Untreated endometrial hyperplasia \\
- Pregnancy \\
- $\quad$ Current VTE or previous idiopathic VTE \\
- $\quad$ Unown CHD \\
- $\quad$ Active liver disease \\
- Porphyria cutanea tarda \\
- $\quad$ Systemic lupus erythematosus \\
\hline
\end{tabular}

are experienced and includes the first 12 months after the last menstrual period. Standard HT regimens are often associated with side effects (e.g. weight gain, irregular bleeds, mood swings) at this time. A meta-analysis of 12 studies in $1998^{368}$ suggested that plasma, as well as urinary, estrogen levels (during both follicular and luteal phases) are, on average, significantly higher in perimenopausal women than young premenopausal women. BMD was shown to decrease rapidly at this time. Reasons for this bone loss, at a time when circulating estrogen levels are not decreased but are high, remain unclear. Whereas irregular bleeds and other "menopausal" symptoms may respond to progestin therapy, appropriate measures to curb bone loss are poorly defined. Standard HT is often poorly tolerated and the estrogencontaining contraceptive pill has been suggested, unless contraindicated. Alternatively, if bone protection is required, non-hormonal bone-active agents may be considered.

In summary:

- HT generally has a favourable benefit/risk ratio if initiated before the age of 60 years or within 10 years of the menopause (window of opportunity)

- It appears that the effects of HT in elderly women and those with established CHD cannot be extrapolated to the young, healthy woman in early menopause.

- HT should only be initiated for specific, proven indications, provided there are no contraindications (see Table $\mathrm{XI}$ ), and should be individualised according to needs.

- HT is indicated in the window of opportunity in subjects with significant vasomotor symptoms, or symptomatic VVA or for the prevention of fractures in individuals at risk It should be considered in all cases of premature menopause at least until age of 51 years

- MHT is not indicated to prevent CHD although some benefit to the cardiovascular system as well as a lowered all cause mortality will be attained if initiated within the window of opportunity

- There are no reasons to place arbitratory time limits on the use of MHT. Duration of treatment should be consistent with the aim of treatment, safety concerns and should be reviewed annualy

- MHT includes a wide range of hormonal products and routes of administration, including tibolone with potentially different risks and benefits. However, evidence regarding differences in risks and benefits between different products is limited.
- The type and route of administration of MHT should be consistent with treatment goals, patient preference and safety issues and should be individualised.

\subsubsection{SERMS, estrogen derivatives, phyto-estrogens, and testosterone}

\subsubsection{Selective estrogen receptor modulators}

SERMs, like raloxifene, lasofoxifene or bazedoxifene, are capable of binding to the estrogen receptor (ER), resulting in estrogen agonist effects in some tissue (e.g. bone) and in estrogen antagonist effects in others (e.g. breast, endometrium). ${ }^{369-379}$ The molecular mechanisms of action of the SERMS are complex and involve differential binding to the ERa (selective partial agonist/antagonist effects) and ERR (selective antagonist effects) receptors, as well as actions which directly decrease the resorptive activity (e.g. decreased production of IL- 6 and TNF- $\alpha$ ) or the number (e.g. decrease in RANKL, increased production of TGF $-\beta_{3}$ ) of osteoclasts. ${ }^{374}$

Raloxifene has been extensively studied and is registered in South Africa for the prevention and treatment of osteoporosis. The drug has estrogen-like effects on bone, lipids and the vasculature, although it differs from standard HT in many ways. Results from the Multiple Outcomes of Raloxifene Evaluation (MORE) study a four-year RCT of 7705 postmenopausal women, showed a very modest increase in spine $(2.6 \%)$ and femoral neck (2.1\%) BMD, and a moderate decrease of around $30 \%$ in the levels of biochemical markers of bone resorption. ${ }^{369,370 ~ A}$ decrease in the rate of vertebral fractures $(50 \%)$, comparable to that of potent ARAs like the bisphosphonates (see below), was, however, documented. There was no difference between raloxifene and placebo groups in the risk of non-vertebral fractures, including hip fractures. These results were corroborated in a recent RCT of more than 10000 postmenopausal women, not selected on the basis of osteoporosis, who participated in the Raloxifene Use for The Heart (RUTH) study. ${ }^{379} \mathrm{~A}$ reanalysis of the MORE data further showed that raloxifene was also effective in significantly reducing the risk of vertebral fractures in subjects with osteopenia, as opposed to osteoporosis. ${ }^{373}$ These results are similar to those obtained with $\mathrm{HT}$ in the WHI and in contrast to the bisphosphonate data, where osteopenic patients without fractures did not show any decrease in the risk of vertebral fractures. No data are available on the effects of SERMs on fracture risk in men.

There is also much interest in the extraskeletal effects of the SERMs. Raloxifene was shown to significantly decrease (HR 0.56; 95\% $\mathrm{Cl}$ 0.38-0.83) the risk of ER-positive invasive breast cancer. ${ }^{369,376}$ Unlike unopposed estrogen, raloxifene does not stimulate endometrial hyperplasia and is not associated with menstrual bleeds or an increased risk of endometrial cancer. Raloxifene has favourable effects on LDL cholesterol and inflammatory markers, and improves vascular endothelial function in postmenopausal women. Although the four-year results from the MORE trial suggested a reduced risk of cardiovascular events in the subset of women with increased cardiovascular risk, ${ }^{363}$ the definitive RUTH study showed no significant effect on the risk of primary 
coronary events. ${ }^{376}$ Raloxifene was, however, associated with an increased risk of fatal stroke (HR 1.49; 95\% Cl: 1.0-2.24) and VTE, although the absolute risk is small ( $<2$ per 1.000 woman-years). Hot flushes are not suppressed by raloxifene and their incidence is, in fact, increased by this drug. Raloxifene may also cause leg cramps.

Bazedoxifene is a SERM not yet approved in South Africa. In an RCT of 6847 postmenopausal women, this drug was not only shown to reduce vertebral fracture risk by $40 \%$, but also caused a $44-50 \%$ reduction in non-vertebral fracture risk relative to placebo in a subset of patients at particular high fracture risk (femoral neck T-score below -3.0 and/or multiple vertebral fractures).

\subsection{Tissue-selective estrogen complex}

The results of a phase III trial employing a combination of various doses of bazedoxifene and CE were recently published. ${ }^{378} \mathrm{CE}$ ( $0.45 \mathrm{mg}$ or $0.625 \mathrm{mg}$ per day) combined with bazedoxifene (20 mg per day) resulted in a significant improvement in BMD at the spine when compared with placebo or raloxifene, and at the hip when compared with placebo. Compared with placebo, it improved vasomotor symptoms and vaginal health without causing endometrial hyperplasia. This tissue-selective estrogen complex (TSEC) compound has the promise of replacing progestins as the endometrial protective agent used in combined HT in non-hysterectomised women. Its use in osteoporosis will be subject to fracture data. It should also be noted that these results should not be extrapolated to other SERM/estrogen combinations, since they all act differently and it is possible that the other available SERMs may not adequately protect the endometrium.

\subsubsection{Tibolone}

The synthetic steroid derivative tibolone has mild estrogenic, progestogenic and androgenic properties, and has been defined as a selective tissue estrogenic activity regulator (STEAR). This drug reduces vasomotor symptoms, may improve mood and libido, and is effective in preventing postmenopausal bone loss. ${ }^{380-382}$ The LIFT trial (Long Term Intervention on Fractures with Tibolone), an RCT of 4,538 postmenopausal women, revealed a significantly decreased risk of both vertebral (HR 0.55 ; 95\% Cl 0.41-0.74) and non-vertebral (HR 0.74; 95\% Cl 0.580.93) fracture. ${ }^{383}$ The tibolone group also had a decreased risk of invasive breast cancer and colon cancer, and no significant increase in the risk of either CHD or VTE. However, the tibolone group had a highly significantly increased risk of stroke (HR 2.2; 95\% Cl 1.14-4.23). This drug should, therefore, not be used in women at risk of stroke. It is also important to note that $1.25 \mathrm{mg}$ of tibolone daily was used in this trial, while $2.5 \mathrm{mg}$ is usually used for vasomotor symptoms.

\subsubsection{Phyto-estrogens}

These preparations are usually isoflavones, lignans or coumestans, and have been shown to improve menopausal symptoms and may also improve lipid profiles and increase BMD. Despite promising earlier reports, ${ }^{384}$ no fracture data are available. These agents cannot, at present, be recommended for the management of osteoporosis.

\subsubsection{Progestins}

As alluded to previously (11.1.2.2), the use of progestins alone can neither be recommended for the prevention, nor treatment, of osteoporosis.

\subsubsection{Testosterone}

The results of genetic (receptor abnormalities) and epidemiologic studies have created a place for androgens in the bone health of females. ${ }^{385}$ Combined treatment with estrogen and testosterone has also been shown to result in significantly higher spine and hip BMD than treatment with estrogen alone. ${ }^{386,387}$ Furthermore, conventional HT with estrogen/progestin is associated with a suppression in gonadotropin levels, which may result in decreased ovarian testosterone production; this could decrease osteoblastic bone formation. It has, in fact, been documented that the addition of testosterone prevents a decrease in serum osteocalcin levels (a marker of osteoblast activity) caused by estrogen treatment. ${ }^{387}$ Selective androgen receptor modulators (SARMs) bind to the androgen receptor with resulting agonist or antagonist activity, and are presently being tested for muscle wasting and osteoporosis.

In postmenopausal women, testosterone administration has been shown to improve libido and mood. Controlled longitudinal studies on the effects of testosterone on serum lipids and cardiovascular morbidity are, however, necessary before this agent can be recommended for the treatment of osteoporosis in postmenopausal females. The American Endocrine Society warns against the use of testosterone in women, since evidence of safety in long-term studies is lacking. ${ }^{388}$

In hypogonadal males, testosterone (e.g. Depo-Testosterone $200 \mathrm{mg}$ intramuscular injection every two to three weeks; threemonthly testosterone undecanoate injections) increases spinal BMD. ${ }^{12}$ Bone formation increases, while resorption may decrease. Treatment should be initiated with small doses. Effects on liver function and the lipid profile should be monitored. Testosterone is contraindicated in patients with prostate cancer. In eugonadal men with osteoporosis, six months of fortnightly treatment with $250 \mathrm{mg}$ depot testosterone resulted in a 5\% increase in spine $\mathrm{BMD}$, without any change in hip BMD. ${ }^{389}$ No fracture data are, however, available in either hypo- or eugonadal osteoporotic subjects treated with testosterone. In general, eugonadal men with osteoporosis are treated with non-hormonal preparations, like the bisphosphonates. Two approaches are followed in the case of hypogonadal men with osteoporosis: some first treat the hypogonadism with testosterone and monitor the BMD response, while others immediately add a bisphosphonate.

\subsubsection{Bisphosphonates}

The bisphosphonates are synthetic pyrophosphate derivatives that contain a carbon atom instead of an oxygen atom, with the resulting $\mathrm{P}-\mathrm{C}-\mathrm{P}$ bond affording resistance to enzymatic and chemical hydrolysis, and creating a class of drugs which avidly 
binds to bone and inhibits resorption. Bisphosphonates are forerunners in the treatment of metabolic bone diseases which are characterised by increased bone resorption, like Paget's disease of bone, myeloma and osteoporosis. ${ }^{390-401}$

\subsubsection{Chemistry, pharmacokinetics and molecular mechanism of action}

The P-C-P structure allows for a great number of chemical modifications, either by changing the two lateral chains on the carbon atom, or by esterifying the phosphate groups. This has resulted in the production of a large number of bisphosphonates with varying activity and duration of action. Binding to bone mineral appears to be largely due to the P-C-P structure, while the antiresorptive activity is a function of the side chains and, therefore, the molecule's three dimensional structure. ${ }^{402,403}$ The potency of the various bisphosphonates in inhibiting bone resorption differs markedly, as indicated in Table XII.

For the first generation, weaker bisphosphonates like etidronate, the dose required to inhibit resorption is high and close to that which also impairs normal mineralisation, while the more potent aminobisphosphonates do not generally cause osteomalacia. The ability of the bisphosphonates to adsorb to bone mineral also contributes to their potency and, particularly, their duration of action. The binding rank order of bisphosphonates has been shown to be: zoledronate $>$ alendronate $>$ ibandronate $>$ risedronate $>$ etidronate $>$ clodronate. ${ }^{404}$ This may explain the apparently more prolonged clinical duration of action of zoledronate and alendronate, compared with the more readily reversible effects of risedronate and etidronate.

The intestinal absorption of bisphosphonates is low $(<1 \%)$, occurs by passive diffusion and is markedly decreased in the presence of food, calcium, juice and tea or coffee. The drug is cleared quite slowly (early half-life of 10 days) and largely $(>50 \%)$ by the skeleton. The rapid intravenous injection of high doses may result in the formation of insoluble aggregates in the circulation, which may impair renal function. The skeletal retention of bisphosphonates is very long (terminal half-life $\geq 10$ years) and, under certain circumstances, even life-long. ${ }^{405}$ The bisphosphonates localise preferentially at sites of bone resorption where mineral is exposed, are internalised by osteoclasts, are buried in bone during the subsequent cycle of bone formation, and become pharmacologically inactive until they are released at a future time during bone remodelling. ${ }^{406}$ The drug is not metabolised and is excreted unchanged in the urine. Renal clearance is high. ${ }^{402}$

The mechanism of action of the bisphosphonates on bone is complex and involves a decrease in osteoclast production, an increase in osteoclast apoptosis and a decrease in osteoclast activity. ${ }^{402-410}$ Of these, the latter predominates and can be ascribed to the specific inhibition of farnesyl pyrophosphate synthase, an enzyme which regulates the biosynthesis of mevalonate in the cholesterol synthesis pathway. ${ }^{403,407}$ This results in the inhibition of protein prenylation and the disruption of key regulatory proteins (e.g. rab, rho, rac) which mediate osteoclast activity. ${ }^{403,407}$ The effects of bisphosphonates on osteoblastic bone formation are even more complex. While some have suggested anabolic effects of bisphosphonates on mesenchymal stem cells, ${ }^{409}$ the prevailing opinion is that formation is inhibited. ${ }^{403-407}$ Whereas some ascribe this merely to the inhibition of resorption and the fact that resorption and formation are generally closely coupled, others have suggested that BPs cause osteoblast apoptosis and inhibit bone formation directly. ${ }^{410}$

Bisphosphonates prevent bone loss in practically all experimental models of osteoporosis, as well as in normal postmenopausal women and in men. ${ }^{390-401}$ Prevention of bone loss is largely explained by a decrease in bone turnover. The depth of osteoclastic resorption cavities is decreased, fewer trabecular microfractures occur and BMD increases by $2-10 \%$, a feature which is most marked in the first year.

The antifracture efficacy of the aminobisphosphonates alendronate (Fracture Intervention Trial, FIT), risedronate (Vertebral Efficiency with Risedronate Therapy, VERT), ibandronate (IBandronate Osteoporosis vertebral fracture trial in North America and Europe, BONE) and zoledronate (Health Outcomes and reduced Incidence with Zoledronic Acid Once Yearly, HORIZON) studies has been extensively documented in more than 30 RCTs. ${ }^{391,401}$ The rate of new clinical and morphometric vertebral fractures was generally decreased by $40-60 \%$, but this figure increased to $90 \%$ in patients with multiple incident fractures. Absolute risk reduction and number needed to treat (NNT) were usually not stated. Protection against non-vertebral fractures was also documented and, although the rate of hip fractures was decreased by $30-50 \%$, this was less convincing, generally confined to patients at high risk of fracture, and sometimes required analysis of pooled data from more than one study.

Studies on the antifracture efficacy of bisphosphonates have been limited to patients at high fracture risk, i.e. subjects with a $\mathrm{BMD}$ in the osteoporosis range ( $\mathrm{T}$-score $<-2.5$ ) or those with prior fracture. In an RCT, risedronate was shown to be ineffective in protecting against hip fracture in postmenopausal women with osteopenia and a BMD T-score above -2.5. ${ }^{87}$ Although a recent post hoc subgroup analysis of pooled data from four different studies claimed that risedronate provided vertebral fracture

Table XII: Potency of bisphosphonates in inhibiting bone resorption

\begin{tabular}{|c|c|c|c|c|c|}
\hline$\sim 1 \mathrm{x}$ & $\sim 10 \mathrm{x}$ & $\sim 100 \mathrm{x}$ & $100-1000 x$ & $1000-10000 x$ & $>10000 x$ \\
\hline etidronate & clodronate tiludronate & Pamidronate & alendronate & $\begin{array}{l}\text { risedronate } \\
\text { ibandronate }\end{array}$ & zoledronate \\
\hline Non-amin & nates & \multicolumn{4}{|c|}{ Aminobisphosphonates } \\
\hline
\end{tabular}

Adapted from Fleisch ${ }^{402}$ 
protection in patients with hip osteopenia, ${ }^{411}$ the antifracture efficacy, particularly at the hip, of the bisphosphonates in subjects with osteopenia (T-score -1.0 to -2.5 ) remains questionable. The lack of convincing long-term fracture data in patients treated with bisphosphonates is also noted. In general, fracture data for three to four years are required for regulatory purposes. Although patients on both risedronate ${ }^{412}$ and alendronate ${ }^{393}$ have been followed up for seven to 10 years, the utility of these studies, which included very small patient numbers $(n<250)$ and no suitable placebo group, to monitor sustained fracture efficacy is limited.

Few head-to-head studies comparing the different bisphosphonates have been performed. These studies have generally compared the magnitude of or the time taken to bring about changes in BMD or biomarkers and, although statistical differences were sometimes found, these were of uncertain clinical importance. ${ }^{276,413}$ A recently published cohort study employing more than 40000 enrollees in large pharmaceutical benefit programmes found no difference in the effectiveness of alendronate and risedronate to prevent non-vertebral fractures. ${ }^{414}$ The retrospective cohort VIBE study (EValuation of IBandronate Efficiency) ${ }^{415}$ found that monthly ibandronate was as effective as weekly alendronate or risedronate in reducing hip fractures, and more effective in reducing the risk of vertebral fractures in adherent subjects, results which require prospective validation. To date, no prospective randomised head-to-head studies comparing the antifracture efficacy of the bisphosphonates have been published. Compliance and adherence is a major issue with any chronic medication in relatively asymptomatic patients, and a number of studies have shown that no more than $50 \%$ of patients are still taking bisphosphonates after one year. ${ }^{416}$

Upper gastrointestinal side effects, including nausea, heartburn, chest pain and vomiting, are the most common adverse effects encountered in clinical practice, and an important reason for discontinuation of treatment. ${ }^{413,417-421}$ Data from RCTs have, however, suggested a much lower incidence $(<1-5 \%)$ of these side effects, which suggests that many patients do not follow the recommendations to take the drug with a full glass of tap water and to not recline afterwards. Earlier reports suggested that daily risedronate was less likely to cause gastrointestinal side effects than alendronate. Randomised, controlled endoscopic studies also revealed fewer oesophageal erosions and gastric ulcers among daily users of risedronate. ${ }^{417,418}$ Recent data from RCTs, like the Fosamax Actonel Comparison Trial (FACT), and systematic reviews have, however, found comparable gastrointestinal side effects between weekly alendronate and risedronate users. ${ }^{413,409,421}$ In general, weekly and monthly dosing schedules of bisphosphonates are less likely to cause gastrointestinal side effects than daily ones. A recent report of oesophageal carcinoma in patients on alendronate emphasised that this drug should not be prescribed to patients with known erosive oesophageal diseases like Barrett's oesophagus, and recommended that bisphosphonate use should be regarded as a possible risk factor for oesophageal cancer. ${ }^{420}$ Subsequent reports have refuted any association between oesophageal cancer and either alendronate or various other bisphosphonates. ${ }^{422}$

Older bisphosphonates, like etidronate, cause mineralisation defects, but the new aminobisphosphonates do not cause osteomalacia. ${ }^{423}$ Rapid intravenous injection of a large dose of bisphosphonate may precipitate acute renal failure, a complication which can be avoided by slow infusion. ${ }^{402}$ Bisphosphonates are not recommended in patients with a creatinine clearance $<30 \mathrm{ml} /$ minute. Intravenously administered bisphosphonates may induce a transient, although occasionally prolonged, severe flu-like syndrome characterised by pyrexia, muscle pain, headache and nausea in $15-45 \%$ of patients. This is usually a first-dose effect, peaks at 24-48 hours, and disappears within three days. It is thought to represent an acute phase reaction, is accompanied by an increase in C-reactive protein (CRP) (although lymphocyte numbers may either increase or decrease), and can be attenuated by paracetamol or non-steroidal anti-inflammatory drugs (NSAIDS). ${ }^{424-426}$ Hypocalcaemia may occur when large doses of bisphosphonates are administered intravenously, particularly in children, and can be avoided by reducing the dosage. Bone pain is sometimes associated with the use of bisphosphonates, but has not been systematically studied and is poorly understood. In an RCT, the intravenous administration of a bisphosphonate has, in fact, been shown to significantly reduce pain in patients with recent vertebral compression fractures. ${ }^{427} \mathrm{~A}$ possible association between serious atrial fibrillation and the use of zoledronate was first reported in the HORIZON study ${ }^{401}$ and later extrapolated to alendronate. ${ }^{428}$ Two recent meta-analyses, however, refuted any association and, after reviewing data on some 20,000 patients treated with bisphosphonates, the FDA also concluded that no significant relationship between atrial fibrillation and bisphosphonates was apparent. ${ }^{429,430}$

The first report of an association between osteonecrosis of the jaw (ONJ) and the use of bisphosphonates was published in 2004. ${ }^{431-436}$ According to the American Association of Oral and Maxillofacial Surgeons, bisphosphonate-related ONJ (BRONJ) is present if (i) exposed bone is present in the mouth for more than eight weeks (the gingival or mucosal tissue surrounding the necrotic bone is usually, but not always, inflamed and sensitive to touch), (ii) a current or previous history of treatment with a bisphosphonate has been obtained, and (iii) there is no history of prior radiation therapy to the jaw. ${ }^{432}$ Early lesions can be demonstrated employing modalities that image bone structure (e.g. panoramic radiographs, CT), bone marrow and soft tissue (MRI), or functional tests (e.g. ${ }^{99 \mathrm{~m} T c-M D P}$ scintigraphy).

A recent review of ONJ noted that, out of 63 cases, more than $80 \%$ were patients with an underlying malignancy (usually myeloma or breast cancer), and $90 \%$ were receiving intravenous bisphosphonates. ${ }^{437}$ In cancer patients administered intravenous bisphosphonates, this relationship appears to be reasonably well established, with a cumulative incidence of $1-12 \%$. Risk factors for the development of BRONJ in these patients include: ${ }^{431-434}$

- Drug-related risk factors (e.g. high dose, potent bisphosphonates and long duration of therapy). It is important 
to note that the dose of bisphosphonates used to treat cancer patients is usually 10 -fold higher than the dose used to treat osteoporosis. A reduction in the dose in oncology practice has already shown that three-monthly administration of intravenous bisphosphonates is significantly safer than monthly administration. ${ }^{438}$

- Dentoalveolar surgery and local oral disease (inflammation, cancer).

- Demographic and systemic factors (e.g. advanced age, renal impairment, obesity and diabetes, smoking, alcohol, concomitant chemotherapy).

- Genetic predisposition.

In patients with osteoporosis treated with oral bisphosphonates, the incidence of $\mathrm{ONJ}$ is thought to be extremely low, in the order of $0.01-0.0004 \% .{ }^{431-434} \mathrm{In}$ a recent medical claims database study of 260000 subjects with osteoporosis, the incidence of ONJ in patients receiving bisphosphonates was similar to that of the general population.$^{439}$

The pathogenesis of BRONJ remains poorly defined. Although bisphosphonates are known to inhibit neoangiogenesis, and earlier reports hinted at a possible association between ONJ and avascular necrosis of the hip, there is little evidence to support an ischaemic basis for the condition. ${ }^{433}$ An association between BRONJ and atypical skeletal fragility resulting from oversuppression of bone turnover (see below) has also been suggested. There is, however, compelling evidence from histologic and radioisotope studies that bone turnover is increased and not reduced within ONJ lesions. ${ }^{433}$ Suggestions that the risk of developing BRONJ can be predicted by assessing systemic bone turnover through the measurement of circulating BTM levels, therefore, have no theoretical basis, nor are they supported by any sound experimental evidence More likely, BRONJ results from bisphosphonate toxicity to bone and/or soft tissue, which is aggravated by infection. . $33,436,440,441^{2}$

Numerous guidelines have been published on the prevention and management of BRONJ. ${ }^{431-433} \mathrm{~A}$ discussion of these guidelines as they relate to oncology patients falls outside the scope of this guideline. With reference to patients with osteoporosis, it is important to note the following:

- The difference in bisphosphonate doses between oncology and osteoporosis patients must be emphasised. Patients and dentists need to be reassured that BRONJ is extremely rare in the osteoporosis setting. In doses approved for osteoporosis, there does not seem to be a difference in the risk of ONJ, whether bisphosphonates are administered orally or intravenously. ${ }^{432}$

- Good oral hygiene and regular dental visits are recommended. It is, however, not necessary to recommend a dental examination prior to starting bisphosphonate therapy for osteoporosis. If major dental surgery is anticipated, it seems prudent to suggest that this be completed before starting bisphophonate treatment.

- In those subjects already receiving a bisphosphonate, dental implant placement/surgery is not contraindicated. Some suggest stopping the bisphosphonate, but there are no data to support this practice. ${ }^{431-433}$ The use of bone turnover markers has been suggested, ${ }^{442}$ but cannot be supported.

- In those subjects with established ONJ, surgical treatment should be conservative, infection should be treated with appropriate antibiotics and pain relief is important, as is referral to an experienced maxillofacial surgeon. Given alternative bone-active agents, it is probably reasonable to discontinue the bisphosphonate.

Atypical fragility fractures (AFFs). In 2000, it was shown that the administration of high doses of the non-aminobisphosphonate etidronate to dogs caused marked suppression of bone turnover, microdamage accumulation and hypermineralisation. ${ }^{443,444}$ This prompted $\mathrm{Ott}^{445}$ to speculate that chronic alendronate therapy in humans might impair bone strength, given the apparent increase in fracture rate with prolonged therapy. In 2005, Pak et al described nine cases of severly suppressed bone turnover (SSBT) with spontaneous non-spine fractures and delayed fracture healing. ${ }^{441}$ Subsequently, no less than eight case reports, four retrospective reviews, and one registry-based national cohort study confirmed a higher prevalence of AFF in patients receiving alendronate. ${ }^{447-451}$ In March 2009, the Medicines and Healthcare products Regulatory Agency (MHRA) in the UK alerted healthcare professionals about this association, and pointed out that product information for alendronate would be updated to include a warning about atypical stress fractures. ${ }^{452}$

A definitive causal relationship between the bisphosphonates and atypical fragility fractures remains to be proven and is, in fact, questioned by many. However, although it probably occurs in a very small minority of patients, there is no doubt that we should take cognisance of this condition, which is largely characterised by the following:

- History of chronic (usually > five years, but may occur earlier) alendronate use. Limited data are available for the other bisphosphonates in support of a causal association with AFF, but this probably merely reflects their lower usage and the limited availability of long-term data.

- AFFs most often involve areas rich in cortical bone (e.g subtrochanteric or diaphyseal femur, pelvic bones), and arise either spontaneously or following minimal trauma.

- Prodrome of pain and tenderness over the impending fracture site.

- Concomitant use of glucocorticoids or estrogen. May be more common in certain populations (e.g. Asians), and in younger subjects ( \pm 68 years) than those who typically present with an osteoporotic fracture.

- Quantitative bone histology shows suppressed bone turnover, similar to the so-called adynamic bone disease found in a subset of patients with chronic renal failure. Serum biomarkers of bone turnover are usually decreased, but often not as markedly as the bone histology. This is an observation that is compatible with previous reports that alendronate may exert more marked suppression (>90\%) on bone turnover at the tissue level, compared with only $50 \%$ reduction from 
baseline in biomarker levels. ${ }^{453,454}$ Microdamage (microcrack quantification) has been shown to be increased in some, ${ }^{455}$ but not all, ${ }^{456}$ studies.

- X-rays may show typical cortical stress fractures, or a simple transverse or oblique fracture of the femur, with beaking of the cortex and diffuse cortical thickening of the proximal femoral shaft.

- Bilateral disease occurs not infrequently. Contralateral pathology often occurs in the same area as the first fracture and may be evident on clinical assessment (e.g. tenderness over the femur shaft), standard radiographs or isotope bone scan.

- History of delayed or absent fracture healing.

Correct management of this syndrome is difficult, given the current state of our knowledge. Clearly, bisphosphonate treatment must be discontinued in the event of an atypical fracture. Contralateral disease should be sought and may require intervention (e.g. prophylactic pinning). Appropriate measures to prevent the development of atypical skeletal fragility include greater awareness of the condition and possibly limiting the duration of bisphosphonate treatment, although no consensus on this issue has been reached (see below).

\subsubsection{Treatment regimens}

\subsection{Bisphosphonate preparations}

Several bisphosphonates are currently registered for the treatment of osteoporosis in this country: daily and weekly oral alendronate with or without vitamin D (branded and generic), risedronate which is available as a weekly or monthly tablet, zoledronate, which is given as an annual intravenous infusion, and ibandronate, which is available as a monthly tablet of 3 monthly injection. Given the poor intestinal absorption of the oral bisphosphonates and their potential to cause upper gastrointestinal irritation, these drugs should be taken on an empty stomach with tap water only and the patient should refrain from reclining after ingestion. It is recommended that calcium and vitamin D intake should be optimised, but these should not be taken simultaneouly with the bisphosphonate.

We have previously expressed concern about differences in the pharmacological properties of generic alendronate preparations from different manufacturers, as well as the lack of proven clinical efficacy of the generic bisphosphonates. ${ }^{457,458}$ Generic bisphosphonates have never been tested for their potency in altering fracture risk or fracture surrogates (BMD, biomarkers). A recent publication by Ringe et $\mathrm{al}^{459}$ again emphasises the superior safety and efficacy of branded alendronate and risedronate compared with generic alendronate, the use of the latter resulting in $40-50 \%$ lower BMD increase, and two- to threefold more gastrointestinal adverse events.

\subsection{Duration and monitoring of bisphosphonate therapy}

The bisphosphonates have revolutionised the management of osteoporosis during the past two decades. Prolonged skeletal retention, the emergence of possible long-term complications like ONJ and AFFs, and uncertainty about antifracture efficacy beyond four years have, however, required a reassessment as to how long patients should ideally be treated with these drugs, and whether a place for a drug holiday exists. Furthermore, adherence to long-term bisphosphonate therapy is notoriously poor, with $45 \%$ of patients being compliant with prescription refills after one year and only $20 \%$ continuing treatment after 24 months. ${ }^{416,460}$

Few studies have addressed the issue of a drug holiday. The Fracture Intervention Trial Long-term Extension (FLEX) was an extension of the FIT study, in which 1,099 patients who had been treated with alendronate for five years were then randomised to receive a further five years of alendronate or placebo. ${ }^{461}$ Subjects at very high risk (e.g. BMDT-score $<-3.5$ ) were excluded. Women who had been switched to placebo lost a statistically significant, but clinically small, amount of bone density, with losses of about 2-3\% more than in those who continued taking alendronate for the full 10 years. Biomarker levels likewise increased in the placebo group, but did not exceed pretreatment values. The risk of hip and morphometric vertebral fracture was reported to be similar between those continuing and discontinuing alendronate, although there was a significantly lower risk of clinical vertebral fractures in alendronate users. The authors concluded that, for many women, discontinuation of alendronate for up to five years does not apprear to significantly increase fracture risk. Results from a post hoc subgroup analysis of FLEX, however, showed that this observation was only true for those women whose BMD at the end of five years was not in the osteoporotic range. The risk of hip fracture was, in fact, increased among individuals in the discontinuation group who had a BMD in the osteoporosis range. ${ }^{462}$ Another study assessed the risk of hip fracture after discontinuation of treatment in women compliant with bisphosphonates for two years, and found the risk to be significantly increased; longer duration ( $\geq$ three years) of bisphosphonate therapy attenuated the increased risk. ${ }^{462}$ In the 3 year extension of the HORIZON study, 1233 patients originally on zoledronate were randomised to receive zoledronate for a further 3 years or to take placebo. Patients continuing with zoledronate had a significantly lower risk of morphometric vertebral fractures but there was no difference in the incidence of hip or non-vertebral fractures between the two groups. The authors concluded that given these findings, the majority of patients could stop zoledronate therapy after 3 years but that consideration should be given to continuing therapy in those patients who are at high risk for vertebral fracture. ${ }^{463}$

It would, therefore, appear that: ${ }^{461,462,464,465}$

- Treatment with bisphosphonates should continue for three to five years depending on the agent being used.

- The fracture efficacy of more than four years of treatment with bisphosphonates requires further evidence-based data.

- The implementation of a drug holiday after three to five years of bisphosphonate treatment is reasonable in those who are not at very high risk of fracture, but must be individualised. In those with high fracture risk, a choice must be made between continuing bisphosphonate treatment and treating with a non-bisphosphonate agent, like strontium ranelate or teriparatide. 


\subsubsection{Denosumab}

Denosumab is not yet registered in South Africa for the treatment of osteoporosis but is available on Section 21 application to the Medicines Control Council.

\subsubsection{Mechanism of action}

Denosumab is a fully human monoclonal antibody to the receptor activator of nuclear factor- $\mathrm{kB}$ ligand (RANKL) that blocks its binding to RANK and thereby inhibits the development and activity of osteoclasts. It is a potent inhibitor of bone resorption. It is administered as a 6 monthly subcutaneous injection.

\subsubsection{Efficacy: effects on BMD, bone turnover and fracture risk.}

The pivotal fracture study (the FREEDOM study) ${ }^{466}$ showed a $68 \%$ reduction in the incidence of new vertebral fractures, a $40 \%$ reduction in hip fractures and a $20 \%$ reduction in nonvertebral fractures after 36 months when compared to placebo. Bone density increased by $9.2 \%$ in the spine and $6 \%$ at the total hip as compared to placeb. Markers of bone resorption (sCTX) were decreased by $86 \%$ at one month and this decrease was maintained over the 36 month duration of the study. Results after 8 years in the FREEDOM extension study showed further significant gains in density and vertebral and non-vertebral fracture incidence remained low. ${ }^{467}$

\subsubsection{Side-effects}

The side effect profile is low. Local skin reactions and exzema have been reported but are extremely rare. In the Phase III clinical studies $1.5 \%$ of patients developed skin infections vs $1.2 \%$ on placebo.

As with other potent anti-resorptives $\mathrm{ONJ}$ and atypical fractures have been reported. Through five years of the FREEDOM extension study eight cases of ONJ and 2 cases of atypical fracture were reported. ${ }^{467}$

As densumab is not excreted through the kidneys it may be a safer treatment option in patients with renal compromise. In these patients adequate calcium intake must be insured to avoid hypocalcaemia.

\subsubsection{NOFSA recommendations on the use of antiresorptive agents}

a. If adequate amounts of calcium (1,200 mg elemental calcium per day) and vitamin $D$ (800-1,000 IU per day) cannot be obtained from the diet, they must be supplemented. The yield of elemental calcium in supplements varies with the calcium salt used (Table X). Calcium carbonate should always be taken with meals to ensure adequate absorption. In general, a dose of $500 \mathrm{mg}$ elemental calcium per day is sufficient. Differences between proprietary preparations of calcium supplements are usually not clinically significant. The prophylactic dose of vitamin D may increase to 2,000 IU/day or more during pregnancy and lactation (see 10.3). Cholecalciferol and ergocalciferol are equipotent, and either may be used as supplement. If $250 \mathrm{HD}$ levels suggest vitamin D deficiency, higher doses may be required (e.g. 50,000 IU every two weeks)

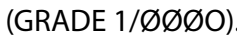

b. HT (including tibolone) generally has a favourable benefit/risk ratio if initiated before the age of 60 years or within 10 years of the menopause (window of opportunity)

- HT should only be initiated for specific, proven indications, provided there are no contraindications (see Table XI), and should be individualised according to needs.

- HT is indicated in the window of opportunity in subjects with significant vasomotor symptoms, or symptomatic VVA or for the prevention of fractures in individuals at risk It should be considered in all cases of premature menopause at least until age of 51 years

- MHT is not indicated to prevent CHD although some benefit to the cardiovascular system as well as a lowered all cause mortality will be attained if initiated within the window of opportunity

- There are no reasons to place arbitratory time limits on the use of MHT. Duration of treatment should be consistent with the aim of treatment, safety concerns and should be reviewed annualy

- MHT includes a wide range of hormonal products and routes of administration, including tibolone with potentially different risks and benefits. However, evidence regarding differences in risks and benefits between different products is limited.

- The type and route of administration of MHT should be consistent with treatment goals, patient preference and safety issues and should be individualized.

c. SERMs, like raloxifene, cannot be regarded as standard firstline treatment for osteoporosis, but have a role in selected cases. For example, consider these drugs when predominantly vertebral fracture protection is sought in subjects at risk of breast carcinoma. Use with caution in the vasculopath at risk of stroke (GRADE 1/ØØØO).

d. Phyto-estrogens, progestins and testosterone cannot be recommended for the sole purpose of fracture protection in women (GRADE 1/ØØØO).

e. In young, hypogonadal men, testosterone replacement should be initiated for non-skeletal benefits. We cannot recommend the addition of a bisphosphonate for skeletal protection right from the start, nor can we advise initial assessment of the skeletal response to testosterone treatment only. We suggest that this decision be individualised, based largely on the severity of the bone disease (GRADE 2/ØOOO).

f. Bisphosphonates should be regarded as first-line treatment for osteoporosis in postmenopausal women, in men and in certain secondary osteoporoses, like GIOP (GRADE 1/ØØØØ).

g. The anti-fracture efficacy of bisphosphonates has been limited to patients at high risk, and should therefore largely be reserved for those with a BMD T-score $\leq-2.5$ and/or a prior fracture (GRADE 1/ØØOO). 
h. Oral bisphosphonates should be taken on an empty stomach with tap water only, and the patient should refrain from reclining. Oral bisphosphonates should not be prescribed to individuals with known upper gastrointestinal disease. Patients administered intravenous bisphosphonates should be alerted to the possible development of a transient, flu-like syndrome, and may require treatment with NSAIDs. Bisphosphonates are not recommended in patients with a creatinine clearance $<30 \mathrm{ml} /$ minute). Although first trimester exposure to bisphosphonates does not appear to pose substantial foetal risk, data are very limited and animal and human studies show that bisphosphonates do cross the placenta. The routine use of bisphosphonates in pregnancy cannot, therefore, be recommended (GRADE 1/ØØØO).

i. Patients, and particularly dentists, must be reassured that, when bisphophonates are used in doses approved for osteoporosis (which are usually $<10 \%$ of those used in oncology), the incidence of osteonecrosis of the jaw is extremely rare and probably no different from that of the general population. A dental examination prior to starting bisphosphonate therapy for osteoporosis is not indicated. If major dental surgery is, however, anticipated, it seems prudent to suggest that this be completed before initiating bisphosphonate therapy. In those subjects already receiving a bisphosphonate, dental surgery is not contraindicated. We do not recommend stopping the bisphosphonate, nor employing a biomarker of bone turnover to aid in such management (GRADE 1/ØØOO)

j. Following three to five years of therapy with a bisphosphonate (depending on the agent used), we suggest that a drug holiday be considered in those who are not at high fracture risk. A recommendation on the duration of such a drug holiday cannot be made, but the patient should clearly be followed up. BMD is usually maintained following the discontinuation of a bisphosphonate, but should be monitored after 18-24 months. In subjects with fractures or a BMD that is still in the osteoporosis range ( $\mathrm{T}$-score $\leq-2.5$ ), in those with ongoing risk factors, and in those whose BMD responded poorly whilst on treatment (e.g. did not increase at all or even decreased), ongoing treatment with a bisphosphonate or with another bone active drug, should be considered (GRADE 1/ØØOO).

k. No clear difference in the antifracture efficacy of the four bisphosphonates registered in this country, alendronate, ibandronate, risedronate or zoledronate, is apparent, and no particular bisphosphonate can be recommended. Until further safety and efficacy data become available, we cannot recommend the use of generic bisphosphonates (GRADE 1/ Ø๐०).

\subsection{Stimulators of bone formation}

ARAs reduce, but do not eliminate, fracture risk and do not restore lost bone structure. Anabolic agents have the potential to significantly increase BMD, restore skeletal microarchitecture and reduce fracture risk to a greater extent than the ARAs. Anabolic agents can increase the number of osteoblast precursors, stimulate the differentiation of these cells into mature osteoblasts, enhance their funcion, or prolong their survival. Few bone formation-stimulating drugs are, however, available. Although fluoride stimulates bone formation, it does not appear to provide fracture protection. Intermittent lowdose PTH administration is presently the only potent bone formation-stimulating agent available. Improved understanding of osteoblast biology in recent years has, however, paved the way to the development of a number of new agents, which are currently being assessed in clinical trials. These include oral secretagogues of PTH (e.g. calcilytics or antagonists of the calcium-sensing receptor (CaSR) in the parathyroid glands, which transiently stimulate endogenous PTH secretion); drugs which manipulate osteogenic factors (e.g. BMP or IGF-1 stimulators); and agents which manipulate osteogenic signalling pathways (e.g. modulators of the Wnt signalling pathway including its natural inhibitors sclerostin, Dkk-1).308,470-472

\subsubsection{Parathyroid hormone}

\subsubsection{Mechanism of action}

Since primary hyperparathyroidism is characterised by bone pain and fractures, it seems counterintuitive to suggest that the administration of PTH may increase BMD and reduce the risk of fractures. However, like glucocorticoids, the action of PTH on bone differs markedly when intermittent, low-dose administration of the hormone is compared with continuous, high-dose exposure. The former is anabolic, whereas the latter is usually catabolic. Intermittent, low-dose administration of intact PTH or its 1-34 fragment, teriparatide, causes rapid stimulation of bone formation by (i) directly stimulating the differentiation of preosteoblasts into mature osteoblasts; (ii) indirectly, by stimulating the production of IGF-1, which also facilitates the differentiation of preosteoblasts to osteoblasts but, in addition, prevents osteoblast apoptosis and enhances the differentiated function of the osteoblast to promote bone formation; and (iii) by down-regulating the Wnt antagonist sclerostin and thereby augmenting the differentiation of preosteoblasts from mesenchymal stem cells. ${ }^{471,472}$ Osteoclastic bone resorption is also increased by PTH but, since this only peaks some 12-24 months later, an "anabolic window" is created which results in

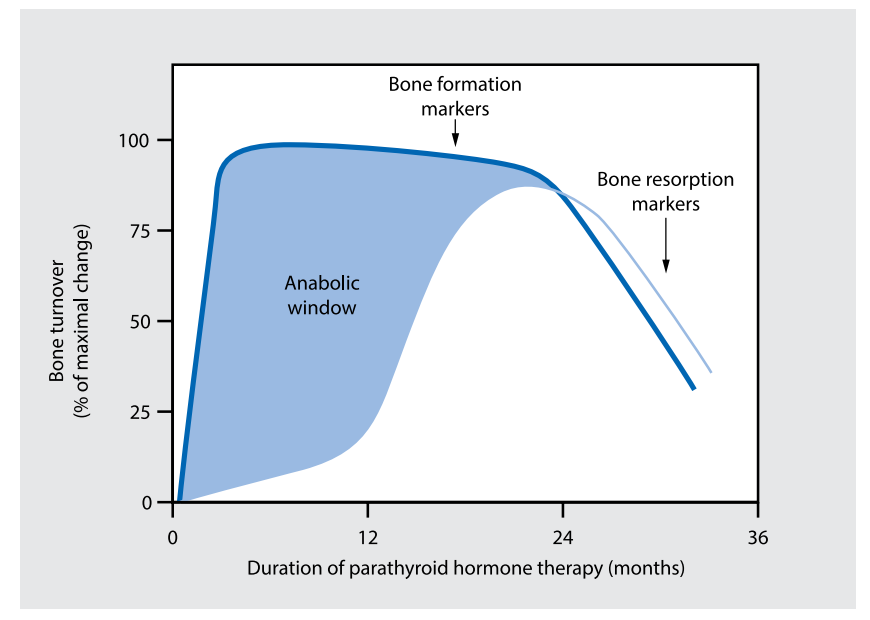

Figure 8: The anabolic window

Adapted from Silverman ${ }^{468}$ and Canalis et $\mathrm{al}^{472}$ 
a significant increase in areal (DXA) and volumetric (QCT) bone mass, size and strength, as well as improvements in trabecular microarchitecture (e.g. increase in trabecular number, thickness and connectivity) (Figure 8).

Several studies have demonstrated either no increase or only a small decrease in areal BMD at cortical sites following the administration of intact PTH or teriparatide. ${ }^{473-476}$ This has been explained by the fact that PTH increases not only BMC, but also bone size (BMD = BMC $\div$ area), although some increase in endocortical porosity has also been documented. The latter is usually self-limiting and, since periosteal apposition is augmented, cortical thickness is maintained and bone strength is not reduced.

\subsubsection{Effects on $B M D$, bone turnover and fracture risk}

The anabolic effects of PTH in humans were first reported by Reeve et al in $1980 .{ }^{478}$ Subsequently, numerous reports have confirmed improvements in BMD, bone remodelling, strength and fracture risk reduction following the administration of PTH/teriparatide. In this guideline, the term PTH without other designation denotes either PTH (1-84) or the hPTH fragment (1-34), teriparatide.

PTH consistently increases aBMD (measured by DXA) in predominantly trabecular bone, like the spine, by $10-15 \%$ over one to three years. ${ }^{471}$ The aBMD in mixed cortical/trabecular sites, like the femoral neck, is usually increased by $1-5 \%$ over comparable time periods, but aBMD changes over mainly cortical sites, like the distal radius, are inconsistent and may decline. ${ }^{471,475,478}$ Volumetric BMD (assessed by QCT) is invariably increased to a greater extent. Treatment with PTH has also been shown to increase spine and hip BMD in men ${ }^{473,476}$ and in women with GIOP. ${ }^{474,479,480}$ Changes in biomarkers of bone turnover can usually be detected within a month after initiating treatment. ${ }^{471,481,482}$ Markers of bone formation increase first, and are followed by an increase in markers of bone resorption (see Figure 8).

Following a number of smaller studies on the effects of PTH on fracture risk, the results of a large RCT, the Fracture Prevention Trial (FPT) involving 1,637 women with postmenopausal osteoporosis, was published in $2001 .{ }^{475}$ Compared with placebo, daily subcutaneous injections of $20 \mu \mathrm{g} \mathrm{hPTH} \mathrm{(1-34),} \mathrm{for} \mathrm{periods}$ as short as 21 months, reduced the risk of new radiographic vertebral fractures by $65 \%$, and non-vertebral fractures by $53 \%$, although separate data for hip fracture prevention are not available. A recent meta-analysis showed that PTH alone or in combination with ARAs reduced vertebral fractures by $64 \%$ and non-vertebral fractures by $38 \% .^{35}$ Using data from the FPT, it was shown that the increase in BMD following teriparatide administration could account for $30-40 \%$ of the antifracture effect of the drug, the majority of the risk reduction resulting from improvements in non-BMD determinants in bone strength. ${ }^{483}$ In a subsequent study, fracture risk reduction was shown to be largely independent of pretreatment bone turnover, although absolute risk reduction was greatest for women with high pretreatment bone turnover. ${ }^{484}$ An RCT which compared the efficacy of teriparatide and alendronate in postmenopausal osteoporosis concluded that teriparatide decreased non-vertebral fractures to a greater degree than alendronate. ${ }^{474}$ Patient numbers in this study were, however, small $(n=73)$, the teriparatide dose used was $40 \mu \mathrm{g} /$ day instead of the recommended $20 \mu \mathrm{g} /$ day, and some of the fractures included in the analysis may have been traumatic in origin. In an 18-month RCT comparing the efficacy of teriparatide with alendronate in 428 women and men with GIOP, teriparatide was not only shown to increase lumbar BMD significantly more $(7.2 \%$ vs. $3.4 \%, p=0.001)$, but also decreased vertebral fracture rate more than alendronate $(0.6 \%$ vs. $6.1 \%$, $\mathrm{p}=0.004)$. The incidence of non-vertebral fractures was not different. ${ }^{277}$ Similar reduced vertebral fracture results have been reported in a $36-$ month extension of the study $(1.7 \%$ vs. $7.7 \%$, $\mathrm{p}=0.007){ }^{485}$

\subsubsection{Indications for the use of teriparatide}

The indications of teriparatide were deliberated by NOFSA and culminated in a position paper which was published in $2004,{ }^{486}$ and recently updated. ${ }^{487}$ Taking due cognisance of anti-fracture efficacy, cost, availability of cheaper drugs, adverse effects and need for daily injections, the following indications for the use of teriparatide, based on cost-effective management principles, are recommended:

i. Male and female patients over the age of 65 years with a BMD T-score $\leq-2.5$, and:

- Two or more fragility fractures; or

- Multiple fragility fractures and an uninterpretable DXA, as first-line treatment and alternative to bisphosphonates to avoid possible blunting of the anabolic PTH response when potent ARAs are used initially (see below).

ii. Patients who have failed treatment with specific bone-active medication, based on:

- Development of new fractures after being on treatment for $\geq 12$ months;

- Unacceptable rate of bone loss (e.g. a decrease in vertebral BMD of $\geq 5 \%$ per annum) as documented on two or more consecutive follow up BMD measurements;

- Intolerance to all other bone-active medication.

iii.Male and female patients on chronic glucocorticoid therapy (three or more months, prednisone equivalent of $\geq 5 \mathrm{mg}$ per day) with:

- BMDT-score $\leq-3.5$, without incident fractures; or

- BMD T-score $\leq-2.5$ with $\geq 1$ fragility fracture; or

- Multiple fragility fractures with bone that cannot be assessed by DXA.

\subsubsection{Side effects and contraindications}

The side effects of PTH are usually limited to occasional nausea, headache and leg cramps. A number of metabolic side effects should, however, be noted. Mild hypercalcaemia occurs in approximately $10 \%$ of patients receiving $20 \mu \mathrm{g}$ teriparatide daily. Clinically significant hypercalciuria is reported to be rare $(<1 \%)$, does not warrant monitoring in most patients, and is 
Table XIII: Contraindications to the use of teriparatide/PTH

\begin{tabular}{|c|c|}
\hline \multicolumn{2}{|r|}{ Absolute } \\
\hline $\begin{array}{l}\bullet \\
\bullet \\
\bullet \\
\bullet\end{array}$ & $\begin{array}{l}\text { Growing individuals (age }<25 \text { years) } \\
\text { Pregnancy and lactation } \\
\text { Pre-existing hypercalcaemia } \\
\text { Renal impairment (serum creatinine }>180 \mathrm{mmol} / \mathrm{L} \text {; creatinine } \\
\text { clearance }<30 \mathrm{ml} / \text { minute) } \\
\text { Marked increase }(\geq 3 \mathrm{x} \text { ) in liver enzymes } \\
\text { Neoplasm(s) in the previous five years } \\
\text { Increased risk of osteosarcoma (e.g. prior skeletal radiation; } \\
\text { Paget's disease of bone) }\end{array}$ \\
\hline \multicolumn{2}{|r|}{ Relative } \\
\hline . & $\begin{array}{l}\text { Mild to moderate renal insufficiency (serum creatinine } 120-180 \\
\mathrm{mmol} / \mathrm{L} ; \text { creatinine clearance } 30-50 \mathrm{ml} / \text { minute) } \\
\text { Moderate increase }(\leq 2 \mathrm{x} \text { ) in liver enzymes } \\
\text { Possible osteomalacia } \\
\text { Previous kidney stones } \\
\text { Gout }\end{array}$ \\
\hline
\end{tabular}

apparently not associated with an increased incidence of renal stone disease. ${ }^{475,488}$ Serum uric acid levels may increase by up to $20 \%$, but clinical gout has not been shown to be more prevalent in patients treated with PTH.

Long-term studies with high-dose PTH, administered to six-weekold Fischer 344 rats, have demonstrated a dose-related increased risk of osteogenic sarcoma. ${ }^{489,490}$ This effect is consistent with lifelong exposure, in a growing rodent, to high-dose PTH, and is unlikely to have relevance to human bone physiology. Shorter or lower dose exposure to PTH has not resulted in the development of osteosarcomas or other bone tumours. All primate studies have failed to show a similar association and osteogenic sarcomas do not occur with increased frequency in patients with primary hyperparathyroidism, nor were they noted in any of the trials performed in many thousands of patients treated with PTH for up to three years. To date, a single case of osteosarcoma has been reported in more than 300000 patients treated wordwide with PTH. ${ }^{491}$ Table XIII lists the contraindications to the use of PTH/teriparatide that have been suggested by NOFSA. 486

\subsubsection{Treatment regimens}

Teriparatide is registered in South Africa for the treatment of osteoporosis, with or without fractures, in postmenopausal women and men. The intact human recombinant molecule, PTH (1-84), is not available in this country.

Standard treatment. A full assessment (see 9.3), with the emphasis on contraindications (Table XIII), should be undertaken prior to initiating therapy. The recommended dose of teriparatide is $20 \mu \mathrm{g}$ per day by subcutaneous injection, for a total duration of 18 months. ${ }^{486,487} \mathrm{~A}$ transdermal patch delivery system has been developed and effectively increases BMD, although fracture data are still awaited. ${ }^{492}$ Serum calcium and uric acid levels should be monitored at one, six and 12 months of treatment, and the dose of supplemental calcium should be adjusted accordingly.

Combination treatment. As PTH increases both bone formation and resorption, it was initially postulated that combining PTH with an ARA would enhance its effect on bone mass and strength. A number of RCTs have yielded somewhat conflicting data:
- In treatment-naïve subjects, combining PTH with a potent ARA, like alendronate, appeared to blunt the anabolic effects of PTH as adjudged by biomarkers of bone turnover, as well as areal and volumetric BMD, in both women ${ }^{493}$ and men $^{494}$ with osteoporosis.

- Concomitant treatment of PTH with less potent ARAs like estrogen or a SERM does not appear to alter the outcome. ${ }^{495}$

- In patients previously treated with ARAs, the anabolic response to PTH depends on the type of ARA, whether a BMD or biomarker response to $\mathrm{PTH}$ is assessed, and whether the ARA is switched or added to the $\mathrm{PTH}$. There is general agreement that the best anabolic response to teriparatide, as adjudged by an increase in BMD and improvements in micro- and macroarchitecture, occurs in bisphosphonate-naïve patients. In those previously treated, the anabolic response to PTH depends on the potency of the ARA that was previously used; e.g. previous etidronate users showed a greater increase in BMD than prior risedronate users who, in turn, showed a greater increase than those who used alendronate..$^{496,497}$ The use of three-month on-off cycles of PTH, whilst continuing alendronate treatment, has also been shown to result in a significant increase in BMD, although not as great as that following daily PTH administration. ${ }^{498}$ Finally, in women treated with ARAs, greater bone turnover increases were achieved by stopping the ARA and switching to teriparatide, whereas greater BMD increases were achieved by adding teriparatide to the ARA. ${ }^{495}$

These results have been interpreted to imply that (i) the best anabolic response to PTH occurs in bisphosphonate-naïve patients, suggesting a role for PTH as first-line treatment in selected patients with severe disease (see 11.2.1.3); and (ii) in patients currently taking ARAs, adding teriparatide rather than stopping the ARA and switching to teriparatide, may confer an improved BMD response. Further studies, employing fracture endpoints, are, however, required before this matter is resolved.

Follow-up treatment. Limited data are available on the skeletal response following discontinuation of PTH treatment. While some earlier studies suggested that BMD is maintained, more recent RCTs, including the European Study of Forsteo (EUROFORS), clearly documented a significant decrease in BMD within the first year following discontinuation of PTH. The decline in BMD following discontinuation of PTH appears to be significantly more rapid in women than in men. ${ }^{499}$ This rapid bone loss can be prevented by initiating treatment with another osteoporosis drug. ${ }^{499-502}$

Retreatment. It remains unclear as to whether a second, discrete retreatment course with teriparatide can produce similar biochemical and BMD changes as seen during the first teriparatide course, since some studies have shown very comparable increases in BMD and in biomarkers of bone formation during retreatment, ${ }^{503}$ whereas others have shown a significantly attenuated response with the second course..$^{504}$ 


\subsubsection{Fluoride}

Fluoride has fallen into disrepute as treatment for osteoporosis largely due to insufficient antifracture efficacy in RCTs. A recent meta-analysis has, however, revealed interesting new data which are briefly reviewed below.

\subsubsection{Pharmacokinetics and mode of action}

Sodium fluoride (NaF) is very efficiently (near 100\%) absorbed from the gastrointestinal tract. Approximately $50 \%$ of the absorbed fluoride is excreted by the kidneys and the remainder is deposited mainly in bone and, to a minimal extent, in other tissues. Skeletal uptake is not homogeneous and proportionately more is taken up by cancellous than by cortical bone. Fluoride affects skeletal tissue by (i) being incorporated into the crystal structure of bone, where it promotes the production of fluorapatite and fluorhydroxyapatite, which are less soluble than hydroxyapatite and may render bone more resistant to osteoclastic bone resorption; and (ii) as a specific mitogen for osteoblasts, stimulates bone formation at the cellular, tissue and organ level. ${ }^{505}$ The molecular mechanism of action of fluoride on osteoblasts remains unclear, although inhibition of phosphatase(s) and augmentation of the mitogen-activated protein kinase (MAPK) signalling pathway, which modulates osteoblast proliferation and differentiation, have been suggested. The response to fluoride treatment is heterogeneous, marked individual sensitivity towards fluoride appears to exist, and approximately one third of subjects do not respond to the administration of fluoride salts at all. ${ }^{506}$ Fluoride also decreases the mineralisation of newly formed bone matrix in a dose-dependent fashion. Higher doses of fluoride may cause frank osteomalacia. This may be related, in part, to inadequate calcium/vitamin D intake or absorption.

\subsubsection{Effects on BMD and fracture rates}

Earlier observational and randomised controlled studies documented a marked increase in spine BMD and a reduction in vertebral fracture rates following fluoride administration..$^{507-510}$ Subsequent RCTs failed to document antifracture efficacy and, in fact, suggested an increased risk of non-vertebral fracture..$^{511-513} \mathrm{~A}$ Cochrane Review and meta-analysis of 11 RCTs published in 2000 concluded that spine BMD was increased by $8.1 \%$ at two years and by $16.1 \%$ at four years of fluoride therapy, while the increase in hip BMD was not significant. ${ }^{514}$ The risk of new vertebral fractures was unchanged at two and four years, as was the risk of non-vertebral fracture at two years; at four years, the risk of nonvertebral fractures was significantly increased. A meta-analysis of 25 RCTs, published in 2008, concluded that there was no significant overall effect on the risk of vertebral or nonvertebral fracture. However, with a daily dose of $\leq 20 \mathrm{mg}$ fluoride equivalents (40 mg NaF/150 mg monofluorophosphate), there was a highly significant reduction in vertebral $(\mathrm{OR}=0.3,95 \% \mathrm{Cl}$ : 0.1-0.9) and non-vertebral ( $\mathrm{OR}=0.5,95 \% \mathrm{Cl}$ : 0.3-0.8) fracture risk. According to these data, low-dose fluoride treatment provided fracture protection comparable to that of PTH or strontium ranelate. $^{35}$
In summary, a single meta-analysis does not provide the basis for making any rational recommendation regarding the antifracture efficacy of fluoride. Moreover, use of this drug is attended by a number of side effects (e.g gastrointestinal, lower limb pain syndrome), which do not necessarily appear to be dosedependent. ${ }^{515}$ Given the very high doses of fluoride employed in previous RCTs (e.g. 75-120 mg NaF per day), the results of future low-dose fluoride studies should, however, be interesting.

\subsubsection{NOFSA recommendations on the use of anabolic agents}

a. We recommend that teriparatide be used in the management of osteoporosis, but only for specific indications. These include subjects with advanced disease (low BMD plus fractures), those who have failed antiresorptive therapy and those with severe GIOP, as detailed in 11.2.1.3 (GRADE 1/ØØØO).

b. Patients considered for teriparatide therapy should be thoroughly assessed (see 9.3), and contraindications (Table XIII) excluded. The standard dose of teriparatide is $20 \mu \mathrm{g}$ subcutaneous per day, for a total duration of 18 months. Serum calcium and uric acid levels should be monitored at one, six and 12 months (GRADE 1/ØØØO).

c. In patients taking HT or a SERM, teriparatide should simply be added to the existing treatment (GRADE 1/ØØØO). With more potent ARAs, like the bisphosphonates, it is less clear whether to switch to teriparatide (i.e. discontinue the bisphosphonate) or whether to add the teriparatide. New data do not convincingly show that prior bisphosphonate exposure causes a blunted anabolic response to PTH. Bisphosphonate washout prior to PTH therapy clearly is unnecessary and does not influence the treatment effect. ${ }^{516,517}$ Current evidence would suggest that the add-on option results in a greater increase in $\mathrm{BMD}$ and is probably the preferred choice. Untill fracture data become available, no firm recommendation on combination therapy can, however, be made (GRADE 2/ØOOO).

d. Following discontinuation of teriparatide treatment, there is a significant decrease in BMD, particularly in women, and treatment with a bisphosphonate, denosumab, strontium ranelate, or a SERM is indicated to preserve the bone mass

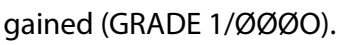

We cannot make a recommendation regarding the feasibility of retreatment once an individual has completed one course of teriparatide. In subjects with severe, fracturing disease, the possibility of another course of teriparatide should, however, not be discounted (GRADE 2/ØOOO).

e. At present, we cannot recommend that fluoride be used in the treatment of osteoporosis, but have taken cognisance of new data on low-dose therapy and await the results of further RCTs (GRADE 1/ØØØO).

\subsection{Drugs with dual or complex actions on bone}

\subsubsection{Strontium ranelate}

The use of strontium in the treatment of osteoporosis dates back over half a century. ${ }^{518}$ Recent clinical trials have emphasised the potential of this agent, which is registered for the treatment of osteoporosis in many countries, including South Africa. 


\subsubsection{Pharmacokinetics and mode of action}

Strontium ranelate is composed of two atoms of the stable nonradioactive trace element strontium, and an organic moiety, ranelic acid. In the gastrointestinal tract, strontium ranelate dissociates into its components: strontium, which influences bone metabolism, and ranelate, which has no pharmacological activity and is eliminated unchanged by the kidneys. ${ }^{519}$ The intestinal absorption of strontium ranelate is rather poor $(25 \%$ bioavailability), and this is reduced by $60-70 \%$ if the drug is taken with food or calcium. Strontium ranelate should, therefore, be taken on an empty stomach.

Preclinical studies suggest that strontium ranelate has a dual mode of action, resulting in the stimulation of boneformation and the inhibition of resorption. Recent animal studies also suggest that strontium ranelate may promote fracture healing. ${ }^{520,521}$ Numerous elegant in vitro studies, largely conducted by Marie and coworkers in France and employing bone cells of rat, mouse, chicken, rabbit or human origin, have demonstrated (i) increased (pre)osteoblast proliferation and differentiation, increased ALP and osteocalcin activity, and enhanced bone collagen synthesis and bone nodule formation following exposure strontium ranelate; as well as (ii) a decrease in the differentiation, activity and lifespan of cultured osteoclasts. ${ }^{522-525}$ The effect of strontium ranelate on osteoblasts is thought to be mediated via the CaSR, since strontium ranelate is known to activate the CaSR which, in turn, activates MAPK and stimulates osteoblast replication. ${ }^{526}$ Osteoclast activity may be inhibited by a direct effect of strontium ranelate on these cells, but new data show that strontium ranelate is capable of downregulating the expression of RANKL while enhancing the expression of OPG in osteoblasts, therefore causing marked inhibition of osteoclasto-genesis. ${ }^{527}$

Animal studies have generally supported the in vitro data. Biomarkers have documented a decrease in resorption, with an increase in the osteoblast marker, ALP. ${ }^{528,529}$ Quantitative bone histology has been less impressive, with some studies demonstrating an increase in bone formation, ${ }^{522,528-531}$ while others reported that bone formation was either not increased, maintained (and not suppressed, as is the case with standard antiresorptive therapy) or only increased following the administration of very high doses of strontium ranelate. ${ }^{532,533}$ Clinical trials, including the pivotal SOTI (Spinal Osteoporosis Therapeutic Intervention) ${ }^{534}$ and TROPOS (TReatment Of Peripheral Osteoporosis Study) ${ }^{535}$ trials, which unequivocally proved the antifracture efficacy of strontium ranelate, yielded similar results. The SOTI trial documented a modest $8 \%$ increase in BSALP and a $12 \%$ decrease in the resorption marker CTX. Biomarkers were not reported in the TROPOS study, but bone biopsies from neither these RCTs revealed significant differences in histomorphometric resorption parameters, nor in the dynamic parameters of bone formation. A modest increase in the mineral apposition rate of subjects receiving strontium ranelate was, however, demonstrated.536 No evidence of a mineralisation defect was noted in either of these trials. In a recent multicentre, open-label, randomised study comparing the effects of strontium ranelate and teriparatide on bone histology and biomarkers in postmenopausal women with osteoporosis, no biochemical evidence of increased bone formation was documented. ${ }^{537}$ Clearly, further studies are required to elucidate the molecular mechanism of action of strontium ranelate in clinical practice. ${ }^{538}$

\subsubsection{Effects on BMD and fracture rates}

Following a number of short-term studies, including the phase II PREVOS (PREVention Of early postmenopausal bone loss by Strontium ranelate), the STRATOS (STRontium Administration for Treatment of OSteoporosis) and the run-in FIRST (Fracture International Run-in Strontium Ranelate Trial) trials, results of the phase III SOTI and TROPOS trials were published in 2004 and 2005. $441,535,539$

The three-year SOTI trial, of 1649 postmenopausal women with osteoporosis and at least one vertebral fracture, documented that $2 \mathrm{~g}$ of oral strontium ranelate daily reduced the risk of new vertebral fractures by $49 \%$ and $41 \%$ after one and three years of treatment, respectively. After three years, lumbar BMD increased by $14.4 \% .{ }^{534}$ The three-year results of the TROPOS trial of 5091 postmenopausal osteoporotic women showed a $16 \%$ reduction in the risk of non-vertebral fractures. Among women at high fracture risk (age $\geq 74$ years and femoral neck BMDT-score $\leq 2.4$ ), the relative risk reduction for hip fracture was $36 \%$. Strontium ranelate increased the femoral neck and total hip BMD by $8.2 \%$ and $9.8 \%$, respectively. ${ }^{535}$ These results have been confirmed in a placebo-controlled five-year study, ${ }^{540}$ while an open-label extension study showed some evidence for sustained antifracture efficacy at eight years. ${ }^{541}$

Although women over the age of 80 years comprise less than $10 \%$ of the postmenopausal population, they contribute more than $60 \%$ of hip fractures. To determine whether strontium ranelate also reduces fractures in the very old, an analysis based on preplanned pooling of data from the SOTI and TROPOS trials included 1488 women, aged 80 to 100 years, followed up for at least three years. In an intention-to-treat analysis, the risk of vertebral and non-vertebral fractures was found to be reduced within one year by $59 \%$ and $41 \%$, respectively. ${ }^{542}$

Women with osteoporosis (BMD T-score < -2.5) contribute only $26 \%$ of hip fractures in the community, whereas most fractures arise from the much larger proportion of women with osteopenia ( $T$-score between -1.0 and -2.5). Although estrogen and SERMs have been shown to be effective, bisphosphonates are generally thought to be rather ineffective to prevent fractures, unless BMD is in the osteoporosis range (see 11.1.3.2). Employing data from the SOTI and TROPOS studies, strontium ranelate was shown to reduce the risk of vertebral fractures by $40-50 \%$ in patients with osteopenia at the spine and/or the hip. ${ }^{543}$ The vertebral antifracture efficacy of strontium ranelate has also been shown to be independent of pretreatment bone turnover. ${ }^{171}$

As alluded to above, strontium ranelate administration results in a significant increase in the BMD of the spine and hip. Because strontium is a heavier element (i.e. has a higher atomic number) than calcium, its incorporation into bone will weaken the penetration of $\mathrm{X}$-rays and, therefore, result in an overestimation 
of measured BMD. ${ }^{544,545}$ During the first year of strontium ranelate administration, this effect is maximal and thought to account for approximately $50 \%$ of the measured change in BMD. It continues to a lesser degree during the second and third year but, thereafter, the strontium content of bone reaches a plateau, so that any further increase can be entirely ascribed to an increase in bone mass. The use of BMD measurements to monitor the antifracture efficacy of ARAs is problematic (see section 12). An excellent correlation between the increase in BMD following strontium ranelate administration and vertebral fracture risk reduction has, however, recently been published: the change in femoral BMD could, in fact, account for $75 \%$ of the antifracture effect of the drug. ${ }^{90,546}$ BMD monitoring during strontium ranelate treatment could, therefore, be valuable to assess both fracture risk reduction and treatment adherence.

\subsubsection{Side effects}

Strontium ranelate is generally very well tolerated, with nausea (7\%), diarrhoea (6\%), headache (3\%) and eczema (5\%) being the most commonly reported adverse events, but only during the first three months of treatment. ${ }^{535}$ Particularly in those with an irritable bowel, it may be advisable to initiate treatment at a lower dose and to gradually increase this over the course of the next two to four weeks.

In pooled data from the SOTI and TROPOS trials, there was an increased risk of VTE in patients treated with strontium ranelate $(0.9 \%$ vs. $0.6 \%)$. The risk of VTE was small and not progressive between year three and five, strontium ranelate has not been shown to have any effects on haemostasis and, when treatment and placebo arms were corrected for subjects with a prior history of VTE, there was no increase in VTE risk in the strontium ranelate arm. ${ }^{547}$ Nonetheless, it is recommended that strontium ranelate be administered with caution in patients at risk of VTE.

During postmarketing surveillance of patients treated with strontium ranelate, cases of DRESS (Drug Reaction with Eosinophilia and Systemic Symptoms) syndrome were reported: less than 20 cases for a total of 570,000 patient-years exposure were documented up to $2008 .^{547}$ This drug hypersensitivity syndrome is, of course, not unique to strontium ranelate and is caused by a large number of commonly used drugs, including antiepileptics and allopurinol. ${ }^{548,549}$ Since systemic involvement (hepatitis, nephritis, endocarditis) can be fatal, it is important to be aware of the association and to discontinue strontium ranelate if any significant skin disorder occurs within two to three months after initiating treatment.

In February 2014 the European Medicines Agency concluded a review on Strontium Ranelate safety (EMA/84749/2014) and published recommendations restricting the use of Strontium Ranelate. The final EMA recommendation was based on analysis of pooled data from randomised studies in approximately 7500 post-menopausal women with osteoporosis. An increased risk of myocardial infarction in patients on Strontium Ranelate as compared to placebo ( $1.7 \%$ vs $1.1 \%$ ) with relative risk of $1.6 \%(95 \% \mathrm{Cl}, 1.07$ to 2.38$)$ and an increased risk of venous thrombotic and embolic events $-1.9 \%$ vs $1.3 \%$ with relative risk of $1.5 \%$ ( $95 \% \mathrm{Cl}, 1.04$ to 2.19$)$ was noted. Data did not show any evidence of increased cardiovascular risk in patients without established, current or past history of ischaemic heart disease or cerebrovascular disease, or in those without uncontrolled hypertension.

Based on these findings the following recommendations were made to healthcare professionals in EU Member States:

- Strontium Ranelate should only be used to treat severe osteoporosis in post-menopausal women and men at high risk of fracture, for whom other treatments for osteoporosis are not possible (contraindications or side effects)

- Strontium Ranelate must not be used in patients with established, current or past history of ischaemic heart disease, peripheral arterial disease and/or cerebrovascular disease, or in those with uncontrolled hypertension

- Doctors should continue to base decisions to prescribe SrR on an assessment of individual patient risk. The patient's risk of developing cardiovascular disease should be assessed before initiating treatment, and on a regular basis thereafter, generally every 6-12 months

- Strontium Ranelate should be discontinued if the patient develops ischaemic heart disease, peripheral arterial disease or cerebrovascular disease, or if hypertension is uncontrolled

- Patients on Strontium Ranelate should be reviewed as necessary

Following a review from the Medicines Control Council an "important medicine safety information" update was sent by Servier Laboratories South Africa to doctors dated 04 April 2014. Titled "New restricted indication from first line to secondline treatment and monitoring recommendations for the use of Protos (strontium ranelate) in postmenopausal osteoporosis".

- These recommendations echoed those of the EMA with one final bullet point - "treatment should only be initiated by a medical practitioner with experience in the treatment of osteoporosis".

\subsubsection{Vitamin D metabolites}

The vitamin D derivatives, calcitriol and alfacalcidol, have been widely studied in postmenopausal women. ${ }^{550-558}$ Doses of calcitriol range from $0.25-1.0 \mu \mathrm{g}$ daily. Alfacalcidol is metabolised to calcitriol via 25-hydroxylation in the liver, and larger doses of this metabolite are generally employed than is the case with calcitriol. These agents stimulate intestinal absorption of calcium, increase serum and urine calcium levels, and decrease PTH and bone resorption. Serum osteocalcin levels increase and there is some experimental evidence that osteoblastic bone formation may be modestly stimulated. ${ }^{559}$ Effects on bone mass and fracture rate have been conflicting and may reflect a narrow therapeutic window, as well as coexistent underlying osteomalacia. There is also some evidence of fall protection. ${ }^{554,557,558}$

Three recently published meta-analyses on the effects of vitamin D metabolites on bone reported widely opposing views. A European study ${ }^{57}$ suggested that the vitamin D metabolites improved lumbar spine BMD and were more effective than native 
vitamin $D$ in preventing vertebral fractures, but did not alter the risk of non-vertebral fracture risk significantly. A Canadian metaanalysis of 16 RCTs concluded that the vitamin D metabolites did not reduce the risk of vertebral fractures, but prevented falls and non-vertebral fractures. ${ }^{558} \mathrm{~A}$ Cochrane Review stated that there is no evidence of advantage of the analogues of vitamin $D$ over parent vitamin D. ${ }^{254}$

The major potential disadvantage of the potent vitamin $D$ analogues is the relatively common occurrence of hypercalcaemia and hypercalciuria when a calcitriol dose exceeding $0.5 \mu$ daily is used. This may result in kidney stones and renal impairment. Concurrent calcium supplementation should be used with care. Clearly, further studies are required in different populations with different calcium intakes before the full potential of the vitamin $D$ derivatives can be determined.

\subsubsection{Anabolic steroids}

Anabolic steroids are analogues of testosterone that promote both protein anabolism and masculinisation. The $17-\beta$ esterified derivatives (e.g. nandrolone) are administered parenterally, while the 17- $\alpha$-methylated steroids (e.g. stanozolol, oxandrolone, danazol) are oral preparations.

Anabolic steroids improve calcium balance, appear to stimulate osteoblast proliferation and inhibit bone resorption. ${ }^{560-563}$ Studies have suggested that at least part of the androgen action on bone may be related to the conversion of androgens to estrogens. ${ }^{563}$ Observational and case-controlled studies have suggested a decrease in fracture risk, but the few prospective RCTs published have been unable to convincingly document that anabolic steroids have any significant antifracture effect. ${ }^{560-562}$ Anabolic steroids decrease fat mass, increase lean body mass (particularly muscle) and improve muscle strength and coordination.

Stanozolol is usualy given continuously at a dose of $5 \mathrm{mg}$ daily. Nandrolone decanoate may be given every four to six weeks at a dose of $50 \mathrm{mg}$ intramuscularly. The use of the anabolic steroids is largely limited by side effects. The $17-\beta$-esterified derivatives, like nandrolone, cause hirsutism and hoarseness of the voice in a dose-dependent fashion which usually, but not invariably, improves when treatment is withdrawn. The 17- $\alpha$-methylated steroids, like stanozolol, increase hepatic transaminase levels in about $50 \%$ of patients (treatment should be discontinued when levels exceed two times the upper limit of the reference range), and may induce an atherogenic lipid profile. Both classes may increase libido and fluid retention.

Anabolic steroids should be reserved for patients with advanced osteoporosis, particularly the frail and elderly in whom the effects on muscle mass, strength and coordination may be most beneficial. Duration of treatment should not exceed 12 months and potential side effects should be carefully monitored.

\subsubsection{Diuretics}

Loop diuretics have been associated with bone loss. ${ }^{564}$ Thiazide diuretics, including indapamide, decrease urine calcium wasting and retrospective analyses of fracture profiles in hypertensive patients have suggested that these drugs may provide fracture protection. No prospective RCT data are available, but a metaanalysis of 13 observational studies, involving some 30000 subjects, revealed that long-term thiazide use was associated with a $20 \%$ reduction in fracture risk. ${ }^{565}$

\subsubsection{NOFSA recommendation on the use of drugs with dual or complex actions}

a. Strontium ranelate should be regarded as a second-line therapy for postmenopausal osteoporosis. Strontium ranelate has also been shown to provide fracture protection in subjects with osteopenia, including the very old ( $>80$ years) (GRADE 1/ $\varnothing \varnothing \varnothing 0)$.

Strontium ranelate should be taken on an empty stomach. In those with an irritable bowel, it may be advisable to initiate treatment at a lower dose. Strontium ranelate is best avoided in those with a previous history of VTE (GRADE 1/ØØØO).

The association, albeit extremely rare, between strontium ranelate and the DRESS syndrome is noted. Strontium ranelate should, therefore, be discontinued if any significant skin rash develops within two to three months of initiating treatment (GRADE 1/ØØØO).

Strontium Ranelate should be prescribed by doctors experienced in the management of osteoporosis. It is contraindicated in those at cardiovascular risk and patients on SR should be regularly reviewed for cardiovascular safety (GRADE 1/ØØOO)

b. The vitamin D metabolites, calcitriol and alfacalcidol, cannot be recommended for the treatment of osteoporosis (GRADE $1 / \varnothing \varnothing 00)$

c. Anabolic steroids have a very small place in the treatment of osteoporosis. In the very old, frail individual with advanced fracturing disease, a short course (e.g. six months) may be considered, largely in an attempt to address the accompanying sarcopenia (GRADE 2/ØOOO).

\subsection{Choice of a pharmacological agent}

Based on the pathogenesis, clinical features, skeletal sites involved and bone turnover, osteoporosis cannot be regarded as a single disease entity and should be viewed as a heterogeneous syndrome. Accordingly, no ideal drug can be recommended for the prevention and treatment of osteoporosis. This is compounded by the lack of adequate head-to-head comparative studies. A recent systematic review of 76 RCTs and 24 meta-analyses, ${ }^{419}$ as well as an assessment of the relative effectiveness of osteoporosis drugs in more than 40,000 enrollees in a pharmaceutical benefit programme, ${ }^{414}$ concluded that data are insufficient to determine the relative efficacy or safety of these agents. Similar to many other chronic degenerative disorders, the choice of pharmacological agent will, therefore, have to be individualised according to (i) the disease profile, (ii) the patient profile, and (iii) available resources and personal preferences. 


\subsubsection{The disease profile (the osteoporosis syndrome)}

The severity of the disease, sites involved (eg. spine vs. hip), causes and risk factors, as well as the rate of bone turnover, may have to be considered before deciding which drug to use.

- If very mild osteopenia without any fractures are present, and no ongoing bone loss seems likely, non-pharmacological measures, coupled with a calcium and vitamin D supplement, and regular follow-up may suffice.

- In osteopaenic patients at increase risk for fracture HT should be considered.

- In subjects with DXA-confirmed osteoporosis (T-score $\leq-2.5$ ), a bisphosphonate or denosumab should be considered.

- In patients unable to take other medication and in whom there are no contraindications strontium ranelate can be used.

- Bone formation-stimulating agents should be reserved for patients with severe osteoporosis, where mere maintenance of bone mass is not sufficient and a sustained increase in BMD is required.

- The choice of drug is also influenced to some extent by the skeletal site(s) involved. Certain agents, like the SERMs, have not been shown to protect against non-vertebral fractures and should, therefore, be reserved for reducing the risk of spine fractures.

- Theoretically, high turnover osteoporosis should respond better to ARAs, whereas a low turnover state may indicate the need for bone formation-stimulating agents. This has, in fact, been shown to be the case with some (estrogen, calcitonin), ${ }^{167,168}$ but not all (bisphosphonates, strontium ranelate), ${ }^{169-171}$ ARAs, and further work is required to validate this hypothesis.

- Specific drugs may be indicated if a particular pathogenetic process is suspected, e.g. gonadal steroids in hypogonadism, and additional vitamin D if accompanying osteomalacia is suspected.

\subsubsection{The patient profile}

Age, general health, concomitant disease, patient preference and the clinical setting in which the patient presents may all have a bearing on the initial choice of drug therapy.
Accompanying disorders may obviously contraindicate the use of certain drugs or favour the use of others. In younger women (50 - 60 years), particularly those with menopausal symptoms, HT should be considered, depending on contraindications and patient preferences. If the patient is at risk of breast cancer, a SERM should be considered. When nothing more than a bonespecific drug is required, a bisphosphonate preparation may be more suitable. In the frail and elderly osteoporotic patient with marked sarcopenia, a short course of anabolic steroids may also be considered.

In men, young premenopausal women and children, evidencebased data on appropriate osteoporosis treatments are scant. These individuals should, therefore, be referred to specialist centres.

\section{(iii) Available resources and personal preferences}

Given the lack of comparative data on the efficacy and safety of osteoporosis agents, decisions on drug selection should be individualised and always attempt to accommodate the preferences of the patient. Therapeutic regimens should always be evidence-based and not rationalised on the basis of dwindling resources.

\subsubsection{NOFSA recommendations on the choice of $a$ pharmacological agent}

1. We acknowledge the fact that, given the heterogeneity of the osteoporosis syndrome and the lack of significant headto-head comparative studies, no ideal drug scenario for management can be recommended.

2. Drug therapy must be individualised, taking due cognisance of the disease profile (particularly the severity of bone loss and skeletal sites involved), the patient profile (age, general health, concomitant disease, clinical setting), and the available resources and personal preferences.

See references online at https://medpharm.tandfonline.com/ toc/ojfp20/current 